\title{
Optimized tomography methods for plasma emissivity reconstruction at the ASDEX Upgrade tokamak
}

\author{
T. Odstrčil, ${ }^{1,2, \text { a) }}$ T. Pütterich, ${ }^{1}$ M. Odstrčil, ${ }^{3}$ A. Gude, ${ }^{1}$ V. Igochine, ${ }^{1}$ U. Stroth, ${ }^{1,2}$ and ASDEX Upgrade Team ${ }^{1, b)}$ \\ 1) Max Planck Institute for Plasma Physics, Boltzmannstr. 285748 Garching, Germany \\ 2) Physik-Department E28, Technische Universität München, Garching, Germany \\ ${ }^{3)}$ Optoelectronic Research Center, University of Southampton, Great Britain
}

(Dated: February 6, 2017)

The soft X-ray (SXR) emission provides valuable insight into processes happening inside of high-temperature plasmas. A standard method for deriving the local emissivity profiles of the plasma from the line-of-sight integrals measured by pinhole cameras is the tomographic inversion. Such an inversion is challenging due to its ill-conditioned nature and because the reconstructed profiles depend not only on the quality of the measurements, but also on the inversion algorithm used. This paper provides a detailed description of several tomography algorithms, which solve the inversion problem of Tikhonov regularization with linear computational complexity in the number of basis functions. The feasibility of combining these methods with the Minimum Fisher Information Regularization is demonstrated, and various statistical methods for the optimal choice of the regularization parameter are investigated with emphasis on their reliability and robustness. Finally, the accuracy and the capability of the methods are demonstrated by reconstructions of experimental SXR profiles, featuring poloidal asymmetric impurity distributions as measured at the ASDEX Upgrade tokamak.

PACS numbers: $52.25 . \mathrm{Vy}, 52.25 . \mathrm{Xz}$

Keywords: Plasma, tomography, tokamaks, Tikhonov regularization

\section{INTRODUCTION}

Elements with a high atomic number Z (e.q. tungsten), are expected to be an essential part of the plasma-facing components (PFC) in future fusion reactors ${ }^{1-3}$. The unavoidable sputtering of these materials caused by plasma wall interactions leads to an influx of the heavy impurities into the plasma. The radiation losses from the partially stripped high- $Z$ ions can be beneficial at the plasma edge because the power exhaust in the divertor is significantly reduced. However, intense radiative cooling in the plasma core leads to a deterioration of the fusion performance ${ }^{4}$. Moreover, unfavorable impurity transport in the plasma core may cause impurity accumulation and, consequently, a radiative collapse of the plasma ${ }^{5}$.

An essential tool for monitoring the spatial impurity distribution are the soft X-ray (SXR) diagnostics. SXR radiation from high- $Z$ elements (i.e. line radiation) is typically much larger than from low-Z elements and main ions (i.e bremsstrahlung). The local impurity density can be estimated from the known relation between the SXR radiation and impurity density as is shown in Refs. 4 and 6 . The spatial information about the SXR radiation is provided by several pinhole cameras surrounding the plasma. Low numbers (i.e. $10^{2}$ ) of available lines of sight (LOS) and the sparse coverage of the plasma leads to a non-trivial, ill-posed inversion problem of the limited angle tomography ${ }^{7}$, i.e. tomography with relatively few cameras.

\footnotetext{
a) Electronic mail: todstrci@ipp.mpg.de.

b) See authors list of U. Stroth et al., Nucl. Fusion 53,104003 (2013)
}

A large number of different algorithms have been proposed for solving the SXR tomography problem. The most common methods are based on a series expansion like the Cormack-Bessel or the Fourier-Bessel ${ }^{8}$, the maximum entropy methods ${ }^{9}$, and the Tikhonov regularization related pixel methods ${ }^{10,11}$. However, the very strong dependence of the tungsten SXR radiation on the electron temperature, along with peaked temperature and peaked tungsten density profiles, results in very steep and localized gradients in the SXR radiation. The presence of such features requires a high dynamic range of the tomographic reconstruction (up to 3 orders of magnitude) in order to resolve the radiation profile from the core to the edge. The best results are typically obtained by employing weakly nonlinear algorithms like the anisotropic Minimum Fisher Information (MFI) ${ }^{12}$ and the Minimum normalized gradient method (MNGR) ${ }^{13}$. A common drawback of the existing algorithms used by pixel based methods is the cubic computational complexity. The improved algorithms described in this manuscript find a solution to this particular inversion problem with linear complexity in the number of pixels. Additionally, various methods for a robust and automatic choice of the optimal regularization level are investigated. The performance of the proposed tomographic methods is verified by reconstructions of the SXR emissivity from a hundred of discharges from the ASDEX Upgrade tokamak (AUG).

AUG is a mid-sized tokamak with $R=1.65 \mathrm{~m}$, $a=0.5 \mathrm{~m}, I_{p} \leq 1.4 \mathrm{MA}, B_{t} \leq 3.1 \mathrm{~T}$, and tungsten covered $\mathrm{PFCs}^{14}$. The metal wall and the high heating power provided by 8 neutral beam injection (NBI) sources delivering up to $20 \mathrm{MW}, 6 \mathrm{MW}$ of ion cyclotron heating, and $4 \mathrm{MW}$ of electron cyclotron heating make this tokamak 
well suited for power exhaust studies relevant for future fusion reactors. The SXR diagnostic in AUG is regularly used for the analysis of the impurity transport ${ }^{15,16}$, magnetohydrodynamic (MHD) instabilities ${ }^{17}$ and fast particles in the plasma ${ }^{18}$. All of these measurements can benefit from the high-quality tomographic methods described in this article.

The current paper is organized as follows: Section 2 contains a description of the AUG SXR diagnostic, which features a progressive design well suited for tomography. In Section 3, we give an overview of the tomography problem and the Tikhonov regularization method followed by the description of the algorithms used in Sec. 4. Various methods for the optimal choice of the regularization level are examined in Section 5. Additionally, since the reconstruction is always subject to experimental errors, a detailed investigation of the variance and the bias is done in Sec. 6. Finally, the performance of the proposed methods on real SXR profiles from AUG is presented in Sec. 7 .

\section{SXR DIAGNOSTIC ON AUG}

The current AUG SXR system has been, with only a few minor changes, operational since the year $2006^{19}$. The diagnostic consists of 15 miniature heads with separate pinholes and chips, assembled to 8 cameras named $\mathrm{F}, \ldots, \mathrm{M}$ resulting in a total of $208 \mathrm{LOSs}$. The cameras $\mathrm{H}$, I and J have three heads each; camera $\mathrm{K}$ has two and cameras F, G, L and M only one head each due to spatial constraints in the divertor and the wall gaps. The design of all heads is identical with a focal length of $14.0 \mathrm{~mm}$ and a slit of size $0.3 \times 5.0 \mathrm{~mm}^{2}$. The arrays of SXR-diodes are centered perpendicularly to the axis of the heads.

Each head is equipped with a linear array of 35 Centronic Series 5T (LD35-5T) diodes of size $4.6 \times 0.96 \mathrm{~mm}^{2}$ with $30 \mu \mathrm{m}$ separation. The diodes are shielded by circular curved Be foils attached behind the slits. The measured foil thickness is $75 \mu \mathrm{m}$ with a relative variability below $5 \%$. The spectral interval with a detection response higher than $50 \%$ ranges from $2.3 \mathrm{keV}$ to about $13 \mathrm{keV}$. To limit the range of the incidence angles of the measured lines of sight, because a too shallow angle is associated with a larger effective thickness and, consequently, a different spectral sensitivity ${ }^{20,21}$, roughly only half of the central diodes on a chip are utilized. Despite this restriction on the incidence angles, the effective thickness of the diodes can vary by $12 \%$ (Cameras H, J) up to $19 \%$ (camera I) between the central and the edge diodes. The increased effective thickness influences the sensitivity only for photons with energies above $7 \mathrm{keV}$, which usually are not emitted in the cold edge plasma observed by the side LOSs. Therefore, this effect was neglected in the calibration.

The positions of all cameras and their observation cones are depicted in Fig. 1. Camera $\mathrm{F}$ is situated in the tokamak sector 5 of 16 , while the rest are in sector

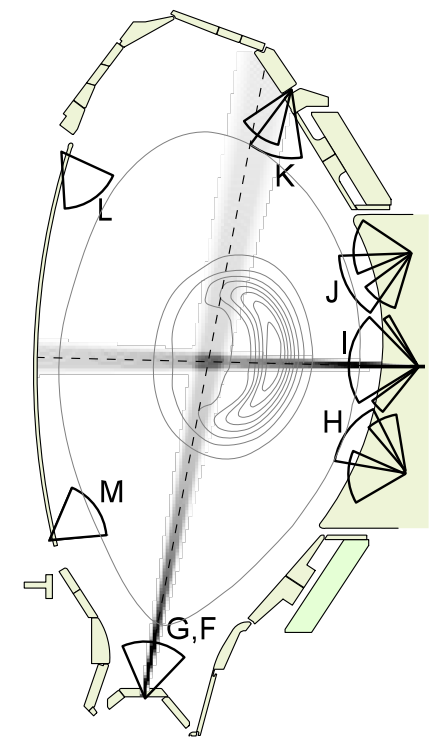

Figure 1. A poloidal projection of the experimental setup of the AUG soft X-ray diagnostic system. The viewing cones of each camera are indicated by black circular sectors. Outboard cameras H, I, J and $\mathrm{K}$ are composed of multiple heads each with the slit in the same position. The viewing geometry of the $\mathrm{F}$ camera is similar to that of the $\mathrm{G}$ camera, however, $\mathrm{F}$ is toroidally separated by $137^{\circ}$ from the other cameras. In gray the volumes of core LOSs from the G and I cameras are shown and in the background, contours of the real SXR emissivity are presented.

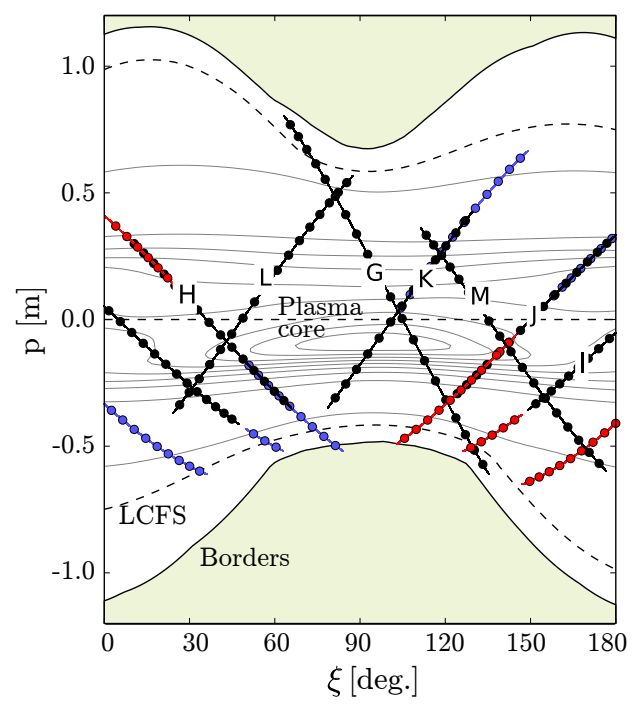

Figure 2. The AUG SXR diagnostic (Fig. 1) depicted in the projection space, where the LOSs are indicated by the dots, $p$ and $\xi$ are the distance and angle of the LOS tangent to the magnetic axis. The LOSs of the central heads are colored black, and the side heads are red and blue. The contours of the Radon transformation ${ }^{22}$ of the radiation profile from Fig. 1 are shown in the background. Beige areas indicate the tokamak vessel, while a typical shape of the last closed flux surface (LCFS) is presented via the dashed line. 
11 separated by $137^{\circ}$. Because camera $G$ has an almost identical geometry as camera $\mathrm{F}$, the toroidal mode number of the emissivity perturbation can be estimated from the SXR measurements alone. The location of the cameras provides an acceptable coverage of the projection space $^{22}$ shown in Fig. 2 with the LOSs from the central head of each camera always crossing the plasma core. The spatial resolution can be estimated from the width of the volume of sights (VOS) in the plasma core. The full width at half maximum of the vertical VOS of camera $\mathrm{F}$ is $7 \mathrm{~cm}$ due to the significant distance from the plasma core, but only $4 \mathrm{~cm}$ for the horizontal camera I, which is the closest. The available spatial resolution of the tomography is moderately improved by synergies of multiple overlapping camera heads as shown in Fig. 1.

The etendue of the LOSs were calculated by a full $3 \mathrm{D}$ model of the VOS including all obstacles in the paths $^{23,24}$. The toroidal spreading of the VOS and the toroidal curvature of the plasma column cause an outward radial shift of the VOS centroid in the plasma core of around $5 \mathrm{~mm}$ compared to the LOS. The largest radial shift due to this effect is observed for the vertical LOSs of the F,G and K cameras. This effect is included in the tomography by an analytical correction ${ }^{23}$.

The signals from the diodes are measured by two distinct acquisition systems. The older one, measuring 80 LOSs (mainly the $\mathrm{H}$ camera and the lower half of the I camera), is equipped with 12-bit analog-digital converters (ADCs) with a $500 \mathrm{kHz}$ sampling frequency and an $80 \mathrm{kHz}$ low pass filter. The newer system acquires the remaining 128 LOSs with 14 bit ADCs, a $2 \mathrm{MHz}$ sampling frequency, and a fixed low pass filter at $500 \mathrm{kHz}$. Both systems are used for the regular tomographic reconstructions and, therefore, the slower system is interpolated onto the $2 \mathrm{MHz}$ sampling rate. The uncertainty of these interpolated signals is increased by factor of 2 to reflect the fact that some temporal information was lost. However, only the new system is usable for studying the fastest MHD phenomena like toroidal Alfven eigenmodes.

The amplification chain is composed of a preamplifier installed close to the tokamak vessel and a two stage main amplifier in a shielded area. The gain of the preamplifier can be varied in the range $3.75-250 \mu \mathrm{A} / \mathrm{V}$ and the main amplifier's gain can be set between 1 and $2^{10}$. The power incident on the diodes is estimated from the general factor $3.62 \mathrm{~W} / \mathrm{A}$ describing the diodes sensitivity in the X-ray range ${ }^{25}$. However, an independent absolute calibration $^{26}$ was not performed. The gain is adjusted before every plasma discharge for each LOSs based on the expected emission intensity. The dark current of the diodes is compensated in the main amplifiers before the discharge starts. Additionally, a small offset is added to use the full range of the ADC without cutting off the noise with negative values. After the acquisition, the raw data is stored in a shotfile database, consuming in total 5.5 GB of disk space per discharge. Finally, because managing of such large data files is very demanding for the memory and computing power, a data edition decimated to $5 \mathrm{kHz}$ is saved as well. It is commonly used for impurity transport and sawtooths studies and the observation of slow MHD modes.

\section{PLASMA TOMOGRAPHY}

\section{A. The tomography problem}

A pinhole camera does not directly measure the local emissivity, but only a signal integrated over the VOS. The goal of the tomography is thus to reveal the best estimate of the local spatial distribution of the emissivity. The power $P_{i}$ incident on the detector $i$ is given by

$$
P_{i}=\iiint_{\mathrm{VOS}} \frac{\Omega_{i}(\mathbf{r})}{4 \pi} G(\mathbf{r}) \mathrm{d} V
$$

where the integration is done over the whole volume observed by the detector; $\Omega_{i}(\mathbf{r})$ denotes the solid angle of the cone from the exposed surface of the detector and $G(\mathbf{r})$ is the emitted power density in $\mathrm{W} / \mathrm{m}^{3}$, i.e. spectral power density integrated over the sensitivity range of the detector. The brightness $b_{i}$ (in units $\mathrm{W} / \mathrm{m}^{2}$ ) can be expressed when both sides of Eq. (1) are normalized by $4 \pi$ over the etendue, defined as the product of the solid angle $\Omega_{i}$ and the area $A$ perpendicular to the axis of the cone $\Omega_{i}$

$$
b_{i}=P_{i} \frac{4 \pi}{\left\langle A \Omega_{i}\right\rangle}=\iint T_{i}(R, z) G(R, z, \varphi) \mathrm{d} R \mathrm{~d} z .
$$

The coordinates $R$ and $z$ correspond to the horizontal and vertical directions in the poloidal tokamak crosssection and $\varphi$ represents the toroidal angle. Since the toroidal extend of the VOS is small, variation of the emissivity in the toroidal direction will be ignored. The operator $T_{i}$ then introduces a mapping of the $2 \mathrm{D}$ emissivity $G$ to a discrete measurement $b_{i}$. This operator is defined as follows:

$$
T_{i}(R, z)=\int \frac{\Omega_{i}}{\left\langle A \Omega_{i}\right\rangle} R \mathrm{~d} \varphi
$$

and describes the geometric properties of the diagnostics. The equation (2) for $b_{i}$ represents the Fredholm integral equations of the first kind, which will be solved by a discretization on a rectangular grid with the total number of pixels $n$. Thus, the problem can be rewritten as a set of $m$ linear equations for $m$ detectors

$$
b_{i}=\sum_{j=1}^{n} T_{i j} g_{j} \quad i \in 1, \ldots, m
$$

$\mathbf{T} \in \mathbb{R}^{m, n}$ denotes a geometry matrix, defining the contribution of a pixel $j$ to the measurement $i$ and $g_{j}$ is the local emissivity at the $j$-th pixel in units of $\mathrm{W} / \mathrm{m}^{3}$. The measured brightness $\hat{b}_{i}$ is subject to experimental errors. Therefore, $\hat{b}_{i}=b_{i}+\xi_{i}$, where $\xi_{i}$ represents a random error with zero mean and a variance of $\sigma_{i}^{2}$. 


\section{B. Tikhonov regularization}

The system of equations obtained by discretization of the Fredholm integral equations is ill-posed and for the tomography of a plasma also usually significantly under-determined $(m \ll n)$. A common method to find a unique and sensible solution is the Tikhonov-Philips regularization ${ }^{27}$ in the general form, which searches for a minimum of the functional $\Lambda(\mathbf{g})$ is given by

$$
\Lambda(\mathbf{g})=(\mathbf{T g}-\hat{\mathbf{b}})^{T} \mathbf{\Sigma}^{-1}(\mathbf{T g}-\hat{\mathbf{b}})+\lambda O(\mathbf{g})
$$

The first term stands for the data fidelity, i.e. the residuum weighted by the expected covariance matrix $\boldsymbol{\Sigma}$, $O(\mathbf{g})$ denotes a regularization functional and $\lambda$ is a positive regularization parameter balancing the strength of a priori constraints with respect to the goodness of fit. For the sake of simplicity, we will further assume that $\mathbf{T}$ and $\mathbf{b}$ are already weighted by a square root of the covariance matrix

$$
\mathbf{T} \leftarrow \boldsymbol{\Sigma}^{-\frac{1}{2}} \mathbf{T} \quad \mathbf{b} \leftarrow \boldsymbol{\Sigma}^{-\frac{1}{2}} \mathbf{b}
$$

The purpose of $O(\mathbf{g})$ is to impose a priori knowledge about the emissivity profiles. This is often some kind of a roughness penalty and a boundary constraint. The regularization operator is typically a quadratic form $O(\mathbf{g})=\mathbf{g}^{T} \mathbf{H}(\mathbf{g}) \mathbf{g}$, with a symmetric and positive semidefinite operator $\mathbf{H} \in \mathbb{R}^{n, n}$ which can be a function of $\mathbf{g}$. A common boundary constraint is zero emissivity at the borders, enforced by adding a sufficiently large positive value to the diagonal points of $\mathbf{H}$ corresponding to the pixels outside of the boundary. Therefore, the regularization operator includes all soft constraints for the solutions. Hard constraints can be imposed by introducing an orthonormal matrix $\mathbf{P} \in \mathbb{R}^{m, l}$ and performing the transformations $\hat{\mathbf{T}}=\mathbf{T P}, \hat{\mathbf{H}}=\mathbf{P}^{T} \mathbf{H P}$ and $\hat{\mathbf{g}}=\mathbf{P}^{T} \mathbf{g}$. The solution will be restricted to a linear subspace generated by the columns of $\mathbf{P}$. An example of $\mathbf{P}$ are the orthonormalized Fourier-Bessel basis functions in the straight field line coordinates.

A minimum of Eq.(5) is now expressed as a quadratic optimization problem for a constant matrix $\mathbf{H}$

$$
\Lambda(\mathbf{g})=\|\mathbf{T g}-\hat{\mathbf{b}}\|_{2}^{2}+\lambda \mathbf{g}^{T} \mathbf{H g}
$$

with the minimum reached at

$$
\mathbf{g}=\left(\mathbf{T}^{T} \mathbf{T}+\lambda \mathbf{H}\right)^{-1} \mathbf{T}^{T} \hat{\mathbf{b}}
$$

A direct inversion of this equation is possible. However, due to the large dimension of the matrices $(n \times n)$, the high computational complexity $\mathcal{O}\left(n^{3}\right)$ of the inversion, and the required number of inversions to a find proper $\lambda$, this procedure is highly impractical.

The optimization problem (6) has a unique solution if the null spaces of $\mathbf{T}$ and $\mathbf{H}$ have only a trivial intersection (i.e. $\operatorname{ker} \mathbf{T} \cap \operatorname{ker} \mathbf{H}=\mathbf{0}$ ). In order to employ computationally efficient numerical algorithms, we will require an invertible $\mathbf{H}$ matrix. Invertibility can always be imposed by a proper definition of the boundary constraints or by adding an identity matrix $\varepsilon \mathbf{I}$ to $\mathbf{H}$, where $\varepsilon$ has the size of the order of the smallest non-zero eigenvalues of $\mathbf{H}$.

\section{Minimum Fisher Information}

The most common regularization operators are the identity operator, suppressing the Euclidean norm of the solution, and the Laplace operator, reducing the curvature of the emissivity profile ${ }^{28,29}$. Nevertheless, the special features of the SXR profiles, like the peaked distribution of the SXR radiation, the sharp gradients, and the large dynamic range, make the nonlinear Minimum Fisher Information regularization (MFI) ${ }^{10}$ an ideal candidate for the regularization functional. This functional can be expressed in the following form:

$$
O_{\mathrm{MFI}}(G)=\iint \mathrm{d} S \frac{1}{G}\left(\nabla_{\mathbf{u}} G\right)^{T} \mathbf{J}_{2}\left(\nabla_{\mathbf{v}} G\right),
$$

where $\mathbf{u}(x, y)$ and $\mathbf{v}(x, y)$ are two locally orthogonal vector fields and $\mathbf{J}_{2} \in \mathbb{R}^{2,2}$ represents a matrix of ones. If $\mathbf{u}$ is parallel with the Cartesian coordinates, the method is called isotropic MFI and if $\mathbf{u}$ is locally tangential to the magnetic flux surfaces, the anisotropic MFI regularization is obtained. More details about the implementation of the anisotropic MFI can be found in Ref. 12. The regularization operator $\mathbf{H}$, representing a linearized and discretized functional (8), is given by:

$$
\mathbf{H}^{(k)}=\sum_{\ell \in\{\mathbf{u}, \mathbf{v}\}} \mathbf{B}_{\ell}^{T} \mathbf{W}^{(k)} \mathbf{B}_{\ell},
$$

where $\mathbf{B}_{\ell}$ denotes a discretized gradient operator $\nabla_{\ell}$ and $\mathbf{W}_{i j}^{(k)}$ is a weight matrix defined as the inverse of $\mathbf{g}^{(k)}$

$$
W_{i j}^{(k+1)}=\delta_{i j} / \max \left\{g_{j}^{(k)}, \varepsilon\right\} .
$$

The MFI regularization must be solved iteratively, because the weight matrix $W$ depends on the emissivity $\mathbf{g}$. The small positive constant $\varepsilon$ prevents division by zero and, moreover, it serves as a weak positivity constraint for the Tikhonov regularization, because the regions with the negative values are strongly smoothed and pushed closer to or above zero. For this work $\varepsilon=10^{-8}$ of the emissivity maximum was used, which is usually an adequate compromise between the positivity and a numerical instability caused by a too low value of $\varepsilon$.

Another method used to enforce the positivity of the solution was proposed in Ref. 30. This algorithm is based on the active set approach from quadratic programming and can be effectively combined with the matrix decompositions presented in the next section.

\section{SPARSE DIAGONALIZATION METHODS}

A large variety of methods were developed to solve the Tikhonov problem, for instance the generalized eigenval- 
ues $(\mathrm{GEV})^{30,31}$ method, the singular value decomposition (SVD) method ${ }^{32}$, the generalized SVD (GSVD) ${ }^{29,33}$ method, bidiagonalization ${ }^{34}$, and the most common is the direct inversion ${ }^{10,12,35,36}$. However, in the current implementation, none of these methods are able to efficiently take advantage of all of the aspects specific to the Tikhonov regularized SXR tomography. First of all, the projection matrix $\mathbf{T}$ has a small numerical rank $r \leq m \ll n$, with typical values of $r=m \sim 10^{2}$ and $n \sim 10^{4}$. The regularization operator $\mathbf{H}$ is a full rank positive definite matrix. Further, both matrices are sparse; the $\mathbf{T}$ matrix for the AUG SXR system has roughly only $5 \%$ non-zero elements and the matrix $\mathbf{H}$ has a regular sparsity pattern and approximately $0.01 \%$ fill-in.

In this section, we present modifications of the SVD and QR algorithms for the solution of the Tikhonov regularization that allows for taking full advantage of these conditions.

\section{A. Sparse SVD decomposition (sSVD)}

Singular value decomposition is a common tool for the solution of the Tikhonov regularization in the standard form, i.e. when the regularization operator is an identity matrix $\mathbf{I}_{n}{ }^{37}$. Hence, it is necessary to transform Eq. (6) into the standard form, before the SVD method can be applied. In the case of $\mathbf{H}=\mathbf{L}^{T} \mathbf{L}$ and if $\mathbf{L}$ is an invertible operator, the transformation is straightforward:

$$
\overline{\mathbf{T}}=\mathbf{T L}^{-1} \quad \overline{\mathrm{g}}=\mathbf{L g}
$$

and then substitution into Eq. (6) we get

$$
\|\mathbf{T g}-\mathbf{b}\|_{2}^{2}+\lambda\|\mathbf{L g}\|_{2}^{2}=\|\overline{\mathbf{T}} \overline{\mathbf{g}}-\mathbf{b}\|_{2}^{2}+\lambda\|\overline{\mathbf{g}}\|_{2}^{2},
$$

where $\|\cdot\|_{2}^{2}$ denotes the Euclidean norm. This transformation method was proposed by Tarasaki ${ }^{32}$ for the discretized Laplace operator. Let's consider a more general case of a sparse and positive definite, but otherwise arbitrary, matrix $\mathbf{H}$. The transformation to the standard form can be performed very effectively by the sparse Cholesky decomposition ${ }^{38}$ of $\mathbf{H}$. A regularized solution is found via the following steps:

1. The sparse Cholesky decomposition of the matrix $\mathbf{H}$ is evaluated through

$$
\mathbf{P} \mathbf{H} \mathbf{P}^{T}=\mathbf{L L}^{T},
$$

where $\mathbf{L}$ is a sparse lower triangular matrix and $\mathbf{P}$ is a fill-reducing permutation.

2. In the next step, Eq. (7) is transformed into the standard form

$$
\left(\mathbf{T}^{T} \mathbf{T}+\lambda \mathbf{P}^{T} \mathbf{L} \mathbf{L}^{T} \mathbf{P}\right)^{-1} \mathbf{T}^{T}=\mathbf{P}^{T} \mathbf{L}^{-T}\left(\mathbf{A}^{T} \mathbf{A}+\lambda \mathbf{I}_{n}\right) \mathbf{A}^{T}
$$

defining the integrated projection matrix $\mathbf{L}^{-1} \mathbf{P} \mathbf{T}^{T}$ as A. Exploiting the sparsity of the $\mathbf{H}$ and $\mathbf{T}$ matrices, such an inversion can be evaluated with $\mathcal{O}(n m)$ complexity. The matrix $\mathbf{A}$ is not sparse anymore, except for the empty rows corresponding to the pixels outside of the plasma boundary. For this reason, the SVD in the following step will be evaluated for non-zero rows only.

3. After the standard form is obtained, the solution can be found by the SVD decomposition of the matrix A, i.e. $\mathbf{A}=\mathbf{U D V}^{T}$. The columns of the matrices $\mathbf{U} \in \mathbb{R}^{m, r}$ and $\mathbf{V} \in \mathbb{R}^{n, r}$ are left-singular and right-singular vectors of $\mathbf{A}$, and $\mathbf{D} \in \mathbb{R}^{r, r}$ is a matrix with singular values on the diagonal in descending order. Substituting the obtained SVD of $\mathbf{A}$ into Eq. (11) results in a decomposition of a form that is already appropriate for the numerical solution

$\mathbf{P}^{T} \mathbf{L}^{-T}\left(\mathbf{A}^{T} \mathbf{A}+\lambda \mathbf{I}_{n}\right) \mathbf{A}^{T}=\tilde{\mathbf{V}}\left(\mathbf{D}^{2}+\lambda \mathbf{I}_{r}\right)^{-1} \mathbf{D} \mathbf{U}^{T}$

where matrix $\tilde{\mathbf{V}} \in \mathbb{R}^{n, r}$ of the reconstruction basis is defined as $\tilde{\mathbf{V}} \equiv \mathbf{P}^{T} \mathbf{L}^{-T} \mathbf{V}$. Nevertheless, since the matrix A is "tall-and-slim", it is usually more economical to calculate an eigendecomposition of the smaller Hermitian matrix $\mathbf{A A}^{T}=\mathbf{X} \mathbf{\Lambda} \mathbf{X}^{T}$ instead of a proper SVD. In such a case $\mathbf{D}=\boldsymbol{\Lambda}^{1 / 2}, \mathbf{U}$ corresponds to the eigenvectors $\mathbf{X}$ and $\mathbf{V}=\mathbf{A}^{T} \mathbf{U} \mathbf{D}^{-1}$. Since the matrix $\mathbf{A} \mathbf{A}^{T}$ has a squared condition number compared to $\mathbf{A}$, one could expect a reduced numerical accuracy. But the regularization is suppressing the least significant eigenvalues and no impact on the reconstruction accuracy was observed.

4. The regularized solution $\mathbf{g}_{\lambda}$ is obtained in the form of a series expansion

$$
\mathbf{g}_{\lambda}=\sum_{i=1}^{r} f_{i, \lambda} \frac{\left(\mathbf{U}_{i}^{T} \mathbf{b}\right)}{D_{i}} \tilde{\mathbf{V}}_{i}
$$

Here $\mathbf{U}_{i}$ and $\tilde{\mathbf{V}}_{i}$ denote columns of matrices $\mathbf{U}$ and $\tilde{\mathbf{V}}$, respectively. The filtering factors are defined as $f_{i, \lambda}=\left(1+\lambda / D_{i}^{2}\right)^{-1}$, monotonously decreasing with $\lambda$ and $i$.

The first step, when the Cholesky decomposition of the $\mathbf{H}$ matrix is performed, has linear complexity because of the regular sparsity pattern and the small number of non-zero elements proportional to $n$. The time spent in this step is negligible. The second step is done by back-substitution of the sparse lower triangular matrix L with $\mathcal{O}(n m)$ complexity. When SVD is replaced by the eigenvector decomposition $(\mathrm{EV})$, then the required number of operations scales as $\mathcal{O}\left(m^{3}+n m^{2}\right)$, while if the thin SVD is applied, the number of flops will increase to $2 n m^{2}+11 m^{3}$ as was shown in Ref. 39, and the measured computing time was increased by a factor of five. The other steps also do not affect the final linear complexity. Moreover, our analysis indicates a cubic dependence of the decomposition time on the number of detectors $m$ in the EV. 


\section{B. Sparse QR decomposition (sQR)}

An alternative method based on a triple application of the QR decomposition was proposed in Ref. 40 by Hosoda. This method does not provide a perfect solution of Eq. (7), however, the solution is a very close approximation, the difference is usually lower than $0.1 \%$. Previous comparisons of the original algorithms ${ }^{32}$ have shown ten times lower computation cost than the former SVD method.

The description of the optimized algorithm for the decomposition of the sparse matrices is summarized below:

1. The first step is identical to the sSVD method; the matrix $\mathbf{A}$ is obtained via the integration of the projection matrix.

2. In the next step, the pivoted rank-revealing $Q R$ decomposition is applied on $\mathbf{A}$

$$
\mathbf{Q}_{1} \hat{\mathbf{D}} \stackrel{R}{R_{R Q R}} \mathbf{A \Pi}
$$

where $\mathbf{Q}_{1} \in \mathbb{R}^{n, r}$ is a unitary matrix, $\hat{\mathbf{D}} \in \mathbb{R}^{r, r}$ is a diagonal matrix with positive diagonal values sorted in non-increasing order, $\mathbf{S}$ is an upper triangular matrix with a unitary diagonal and $\boldsymbol{\Pi}$ is a permutation matrix. The Q-less QR decomposition can be employed, because $\mathbf{Q}_{1}$ is not required explicitly in the following steps.

3. The ordinary QR decomposition is applied to the matrix $\mathbf{\Pi} \mathbf{S}^{T}$

$$
\mathbf{Q}_{2} \mathbf{R}_{2} \stackrel{Q R}{=} \Pi \mathbf{S}^{T}
$$

where $\mathbf{R}_{2} \in \mathbb{R}^{r, r}$ is an upper triangular matrix and $\mathbf{Q}_{2} \in \mathbb{R}^{m, r}$ is a unitary matrix.

4. Now the diagonal matrix $\mathbf{D}$ is swapped with the $\mathbf{R}_{2}$ matrix

$$
\mathbf{M}=\hat{\mathbf{D}} R_{2}^{T} \hat{\mathbf{D}}^{-1}
$$

and the third QR decomposition of the matrix $M$ is computed

$$
\mathbf{R}_{3} \mathbf{D}_{R} \mathbf{Q}_{3} \stackrel{Q R}{=} \mathbf{M}
$$

Just like in the second step, the Q-less QR decomposition can be used. $\mathbf{R}_{3} \in \mathbb{R}^{r, r}$ is an upper triangular matrix with a unitary diagonal and $\mathbf{D}_{R} \in \mathbb{R}^{r, r}$ is a diagonal matrix.

5. In the last step, the final integration by the $\mathbf{L}$ operator is performed and the decomposition is transformed to the form analogous to the preceding

$$
\begin{aligned}
\operatorname{method} & \\
\mathbf{R} & =\hat{\mathbf{D}} \mathbf{R}_{3}^{T}\left(\mathbf{D D}_{R}\right)^{-1} \\
\mathbf{D} & =\hat{\mathbf{D}} \mathbf{D}_{R} \\
\mathbf{U} & =\left(\mathbf{R}_{3}^{-1} \mathbf{Q}_{2}^{T}\right)^{T} \quad \text { (Forward-substitution) } \\
\mathbf{V} & =\mathbf{A U D}^{-1} \\
\hat{\mathbf{V}} & =\left(\mathbf{P L}^{-T} \mathbf{V}\right)^{T} \quad \text { (Back-substitution). }
\end{aligned}
$$

Since the definitions of the matrices $\mathbf{U}, \mathbf{D}$ and $\hat{\mathbf{V}}$ are now consistent with the notation in the sSVD algorithms, the regularized solution can be estimated by formula (12). The arguments used for the analysis of the computational complexity of the SSVD method are valid here as well. In the sQR algorithm the most expensive step is the first RRQR decomposition requiring $\mathcal{O}\left(n m^{2}+m^{3}\right)$ operations $^{39}$. The other steps have a $\mathcal{O}(\mathrm{nm})$ complexity or even lower. Since the asymptotic complexities of both algorithms are identical, it is necessary to assess these algorithms by a direct comparison of the computation time.

\section{Computational effort}

In addition to the algorithms introduced in the previous section, also the GEV method $^{30}$ based on the GEV routine for sparse Hermitian matrices from the ARPACK library $^{41}$ and GSVD ${ }^{33}$ were included in the investigation in this section, because both are regularly used for the solution of the generalized Tikhonov problem. The comparison was performed for a variable number of pixels $n$ and with a fixed number of $m=k=200$ detectors. The number of the non-zero elements in the projection matrix was about $5 \%$ and the anisotropic regularization operator used had all non-zero elements regularly aligned in nine diagonals. All computation tests were run in a single thread on an Intel Core i5-2540M processor to allow a fair comparison of these methods. An algorithm based on the direct inversion of the Tikhonov problem by the sparse Cholesky solver ${ }^{12}$ was used as reference method. The measured computation times are shown in Fig. 3 with dashed lines indicating the asymptotic complexity.

The fastest method is based on the sQR decomposition and it takes about $34 \mathrm{~ms}$ for a moderate resolution $40 \times 60$ pixels. sQR is followed by the sSVD algorithm, which is $30 \%$ slower. Both algorithms show a linear time complexity in the number of pixels $n$, which tails off for $n \lesssim 5 m$. Other algorithms based on the GEV and the GSVD are, for a reasonable range of $n$, significantly slower than the direct inversion. The time measured for the algorithm based on the sparse GEV are have a quadratic complexity in $n$, but with a rather large multiplication factor. Finally, GSVD based on sine-cosine decomposition $^{42}$ is not able to efficiently take advantage of the matrix sparsity and, thus the computing time scales as $\mathcal{O}\left(n^{3}\right)$. The decomposition methods even further outperform the direct solvers because at least $3-10$ 
inversions are necessary to solve the inner loop of the MFI algorithm and find the optimal regularization parameter. But, once the decomposition is available, the inner loop can be solved almost instantaneously in $\approx 1 \mathrm{~ms}$ with $\mathcal{O}(m k)$ complexity. The numerical accuracy of all algorithms solving Eq. (7) was better than $10^{-12}$, except for sQR, which provides inversion with a difference of $10^{-3}$. This is still well below the experimental uncertainties.

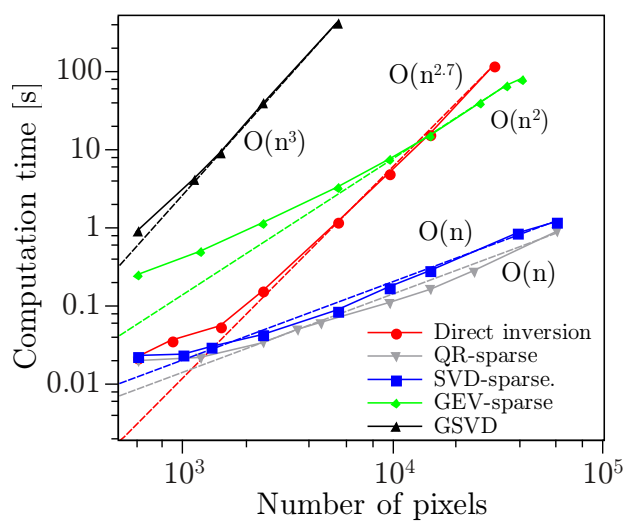

Figure 3. Computation times of different decomposition methods were compared to the direct inversion algorithm based on the sparse Cholesky decomposition ${ }^{12}$. The asymptotic complexity is indicated by the dashed lines.

The sSVD and sQR decomposition methods are very efficient especially in the case of a low-rank projection matrix. The direct inversion is faster only if the rank $r$ is comparable with $n$ or at very low resolution $n \ll 10^{3}$ as was shown in Refs. 43 and 44 . Since the large scale changes in the emissivity profile usually evolve on much slower timescales than the sampling frequency, it is not necessary to evaluate a decomposition for each time frame separately, but instead the discharge can be divided into short blocks reconstructed at once. In such cases, it is possible to compute roughly 200 frames/s at a moderate resolution of $40 \times 60 \mathrm{px}$.

\section{THE OPTIMAL CHOICE OF THE REGULARIZATION PARAMETER}

One of the key issues related to the Tikhonov regularization is the proper selection of the regularization parameter. The optimal parameter $\lambda_{\text {opt }}$ minimizes the difference between the reconstructed and the original profile. Since the original radiation distribution is not available in the experiment, this parameter must be determined purely from the measurements and a priori knowledge. Moreover, the optimal estimator of $\lambda_{\text {opt }}$ should be robust, reliable, and the computation complexity should be polynomial in $m$, independent of the number of pixels $n$. Hence, the optimal solution must be found without the explicit evaluation of $\mathbf{g}_{\lambda}$.

Many different methods have been proposed for the choice of the regularization level. Commonly used methods include the Morozov's discrepancy principle (DP $)^{7,45}$, the curvature of the L-curve ${ }^{46}$, the generalized crossvalidation $(\mathrm{GCV})^{28,32}$, the predicted sum of squares $(\mathrm{PRESS})^{47}$, the corrected Akaike information criterion $\left(\mathrm{AIC}_{\mathrm{C}}\right)^{48}$ and the quasi-optimality criterion ${ }^{49,50}$. However, with the exception of DP, these methods are not regularly used in SXR plasma tomography. Since the behavior of these methods significantly depends on the investigated problem, the only way to identify the most suitable one is to perform a comparison on artificial and experimental measurements. Tests of these methods on the artificial radiation profiles allowed for identification of the most promising methods, namely PRESS, $\mathrm{AIC}_{\mathrm{C}}, \mathrm{GCV}$, and DP. Due to the highly ill-posed character of SXR tomography, the corner of the L-curve is insignificant or missing on real measurements. The quasi-optimality criterion is not able to provide a single, reliable and unique optimum even for simple artificial profiles. Finally, the compatibility of the remaining methods with the nonlinear MFI needs to be investigated.

The regularization level in the following section will be quantified by the quantile $q_{\lambda}$ in the set $\left\{D_{i}^{2}\right\}$ equal to $\lambda$. Compared to the regularization coefficient $\lambda$ itself, whose value does not have a direct meaning and depends on the normalization of the $\mathbf{H}$ and $\mathbf{T}$ matrices, $q_{\lambda}$ roughly corresponds to the fraction of degrees of freedom removed by the regularization. A value close to one results in very high regularization, almost completely ignoring the measurements, while a value of about zero causes negligible regularization.

\section{A. Discrepancy principle}

For the choice of the optimal regularization parameter, the Morozov's discrepancy principle (DP) is the most common method used by plasma physicists ${ }^{11,12,36,43,45}$. The regularization parameter is chosen such that the residual norm for the solution $\mathbf{g}_{\lambda}$ satisfies

$$
\left\|\mathbf{T g}_{\lambda}-\mathbf{b}\right\|_{2}^{2}-\|\boldsymbol{\epsilon}\|_{2}^{2}=0
$$

where $\boldsymbol{\epsilon}$ is the estimated noise level. This condition together with the minimization of the regularization functional leads to a constrained quadratic optimization problem, easily solvable by an iterative root-solver. A unique solution satisfying the condition (14) exists if $\left\|\mathbf{b}-\mathbf{U U}^{T} \mathbf{b}\right\|_{2}<\|\boldsymbol{\epsilon}\|_{2}^{2}$ and $\|\mathbf{b}\|_{2}^{2}>\|\boldsymbol{\epsilon}\|_{2}^{2}$, which is always fulfilled if the projection matrix $\mathbf{T}$ has full rank and the measurements exceed the noise level. For the decomposition methods introduced earlier, the residuum can be expressed in the following form

$$
\left\|\mathbf{T g}_{\lambda}-\mathbf{b}\right\|_{2}^{2}=\sum_{i=1}^{m}\left(\left(1-f_{i, \lambda}\right) \mathbf{U}_{i}^{T} \mathbf{b}\right)^{2}
$$

where $f_{i, \lambda}$ are the filtering factors introduced in Eq. (12), $f_{i, \lambda}=0$ for $i \geq r$ and $\mathbf{U}$ must be multiplied by the matrix 
$\mathbf{R}$ from the right for the sQR method. The root in $\lambda$ can be found with a mere $\mathcal{O}(m k)$ complexity. The choice of Eq. (14) was motivated by the fact that if the Tikhonov regularization is correctly weighted by the statistical uncertainties, while an approximately normal noise distribution is assumed, then the residuum will have a $\chi^{2}$ distribution with $p$ degrees of freedom. The expected value of $\chi^{2}$ is also $p$ and it can easily be checked that $p=m-k+\sum_{i=1}^{k}\left(1-f_{i}\right)^{2}$. Since the existence of the root is not generally guaranteed, we assume $p \equiv m$, which leads to Eq. (14) and tends to a slight overestimation of the regularization parameter.

The discrepancy principle strongly depends on a reliable estimate of the uncertainty level for each signal and time frame and such knowledge is usually not directly available. We have estimated the statistical variance from the temporal and spatial characteristics of the measured signals. However, the systematic errors are unknown, and they can also depend on the profile or the energy spectrum of the radiation. For these reasons, different methods depending solely on the measured data must be considered as well.

\section{B. PRESS - Predicted residual error sum of squares}

The method called predicted residual error sum of squares (PRESS) ${ }^{51}$ is based on the basic leave-one-out cross-validation. The model minimizing the PRESS score should have the best predictive capability. Over-fitted models tend to fit noisy features in the data, reducing their predictive capability. On the other hand an overregularized model is not able to follow real features in the measurements, leading to an increase of the PRESS as well. The value of the PRESS score can be expressed by the following formula

$$
\mathcal{P}(\lambda)=m^{-1} \sum_{l=1}^{m}\left[\left(\mathbf{T g}_{\lambda}^{(l)}\right)_{l}-b_{l}\right]^{2}
$$

where $\mathbf{g}_{\lambda}^{(l)}$ is the solution in which the $l$-th detector was removed. A simplified solution was found for the decomposition based methods ${ }^{52}$

$$
\mathcal{P}(\lambda)=\frac{1}{m} \sum_{k=1}^{m}\left(\frac{\sum_{j=1}^{r} \sum_{l=1}^{m}\left(1-f_{j}\right) U_{k j} U_{l j} b_{l}}{\sum_{j=1}^{r}\left(1-f_{j}\right) U_{k j}^{2}}\right)^{2}
$$

and the optimum can be found with $\mathcal{O}(m r)$ complexity. The first comparison of the PRESS method and the GCV method, presented by Iwama ${ }^{47}$, showed the superiority of the GCV, because if the hat matrix

$$
\hat{\mathbf{A}}_{\lambda} \equiv \mathbf{T}\left(\mathbf{T}^{T} \mathbf{T}+\lambda \mathbf{H}\right)^{-1} \mathbf{T}^{T},
$$

is close to diagonal, the PRESS method is not able to provide a reliable estimate due to a lack of redundancy in the model. But this is not the case for SXR tomography, where the assumption of smoothness and a large number of overlapping LOSs guarantee sufficient redundancy.

\section{GCV - Generalized cross-validation}

The underlying principle in the generalized crossvalidation $(\mathrm{GCV})$ is very similar to the PRESS method. GCV was first introduced by Wahba ${ }^{53}$ and further developed by Golub ${ }^{54}$ for the ridge regression and used for plasma tomography by Iwama ${ }^{28,55}$ later. The GCV score is defined as

$$
\mathcal{G}(\lambda) \equiv \frac{\left\|\left(\mathbf{I}_{m}-\hat{\mathbf{A}}_{\lambda}\right) \mathbf{b}\right\|_{2}^{2}}{\operatorname{Tr}\left(\mathbf{I}_{m}-\hat{\mathbf{A}}_{\lambda}\right)^{2}}
$$

Using a decomposition (12) we obtain

$$
\mathcal{G}(\lambda)=\frac{\left\|\mathbf{T g}_{\lambda}-\mathbf{b}\right\|_{2}^{2}}{\left(r-\sum_{i=1}^{r} f_{i}\right)^{2}}
$$

and the residuum in the numerator is evaluated from Eq. (15). The existence of a global and unique optimum of the GCV curve is not guaranteed. Consequently, we have observed on real measurements that the proper minimum is not the global one, but, if it was present at all, the local minimum with the highest regularization.

\section{D. $\quad \mathrm{AIC}_{\mathrm{C}}$ - Corrected Akaike information criterion}

An alternative way of selecting the optimal regularization parameter is a negative entropy minimizing principle called the corrected Akaike information criterion $\left(\mathrm{AIC}_{\mathrm{C}}\right)$, originally proposed by Hurvich in Ref. 48. $\mathrm{AIC}_{\mathrm{C}}$ is a modification of the well known $\mathrm{AIC}^{56}$ method corrected for finite size samples. $\mathrm{AIC}_{\mathrm{C}}$ is defined by the following formula:

$$
\mathcal{A}_{c}(\lambda)=\left\|\mathbf{T g}_{\lambda}-\mathbf{b}\right\|_{2}^{2}+m(\ln (2 \pi)+1)+\frac{2(p+1) m}{m-p-2}
$$

where $p$ denotes the number of parameters. The effective number of parameters of the Tikhonov regularization is determined as $^{57} p=\operatorname{Tr}\left(\hat{\mathbf{A}}_{\lambda}\right)=\sum f_{i}$. Because $\mathcal{A}_{c}(\lambda)$ is not a homogeneous function of the residuum, the position of the minimum will depend on the estimated absolute level of the noise. This means that under real experimental conditions a systematic error may be introduced.

\section{E. Effect of the nonlinear MFI iterations}

Since the quality of the sQR and the SSVD method is almost the same, all further reconstructions will be performed only by the sSVD method. The convergence and the final solution of the MFI depend also on the choice of the regularization parameter in the intermediate steps. The decomposition $\left\{\mathbf{U}^{(\mathbf{i})}, \mathbf{D}^{(\mathbf{i})}, \mathbf{V}^{(\mathbf{i})}\right\}$, obtained in each iteration by the SSVD or the sQR method, is better adapted to describe the solution than the previous one. This is illustrated by the reconstruction of a rather peaked phantom (artificial profile) in Fig. 4, where the 
optimal GCV reconstruction is shown in the upper row and the energy spectrum of the $i$-th iteration defined as $\left(\mathbf{U}^{(\mathbf{i})} \mathbf{b}\right)_{j}^{2}$ is in the lower row. In the 0-th MFI iteration, equivalent to the 1st order Tikhonov regularization, the energy spectrum is broad, the signal is mixed with the noise and separation of the dimensions dominated by the random variability is possible only for $i>150$. However, in the first and the second iteration the spectrum is significantly compressed, and the dimensions dominated by the noise can now be clearly separated for $i>70$.

The effects of the MFI iteration on different regularization selection methods is summarized in Tab. I. The first column contains the regularization level for each iteration described by $q_{\lambda}$ and the second column is the relative deviation of the tomogram $\mathbf{g}_{\lambda}$ from the known phantom g:

$$
\delta=\left\|\mathbf{g}_{\lambda}-\mathbf{g}\right\|_{2} /\|\mathbf{g}\|_{2}
$$

Clearly, the optimal regularization level increases with each iteration, while $\delta$ decreases for all methods. At the same time, the optima for the GCV, PRESS, and $\mathrm{AIC}_{\mathrm{C}}$ methods becomes more pronounced and easier to distinguish due to the better signal/noise separation in the energy spectrum (Fig. 4). The GCV and the PRESS methods provide the best estimates of the regularization level with the final relative deviation $\delta$ only higher than the optimum by $1 \%$. The discrepancy principle and the $\mathrm{AIC}_{\mathrm{C}}$ method have both selected slightly over-regularized solutions.

Moreover, we have observed that using all of the methods described above in the intermediate iteration steps leads to a significant increase in the failure rate of the tomography on the experimental signals and the convergence is slowed down. Therefore, we have set the regularization to a fixed value $q_{\lambda}=75 \%$, which is above the typical regularization level in AUG and the optimal regularization is selected only in the last iteration.

Table I. The performance of different regularization selecting methods on the peaked radiation phantom shown in Fig. 4 during MFI iterations. Artificial noise of $5 \%$ was added to the measurements. The value of the optimum regularization was determined as the position of the minimum deviation between reconstruction and the phantom. The regularization level is described by $q_{\lambda}$ defined as a quantile of the set $\left\{D_{i}^{2}\right\}$ being equal to $\lambda$.

\begin{tabular}{|c|c|c|c|c|c|c|}
\hline \multirow[b]{2}{*}{ Method } & \multicolumn{2}{|c|}{0. step } & \multicolumn{2}{|c|}{ 1. step } & \multicolumn{2}{|c|}{ 2. step } \\
\hline & $\overline{q_{\lambda}(\%)}$ & $\delta(\%)$ & $\bar{q} q_{\lambda}(\%)$ & $\delta(\%)$ & $\overline{q_{\lambda}(\%)}$ & $\delta(\%)$ \\
\hline optimum & 4 & (12) & 15 & $(8)$ & 37 & $(7)$ \\
\hline $\mathrm{GCV}$ & 13 & 13 & 37 & 10 & 56 & 8 \\
\hline PRESS & 28 & 21 & 38 & 10 & 52 & 8 \\
\hline $\mathrm{DP}$ & 22 & 18 & 54 & 14 & 76 & 12 \\
\hline $\mathrm{AIC}_{\mathrm{C}}$ & 12 & 22 & 56 & 14 & 71 & 11 \\
\hline
\end{tabular}

Table II. Performance comparison of different regularization methods on various artificial SXR profiles. The score was measured as a relative deviation [\%] with respect to the phantom (Eq. (19)). The first line corresponds to the ideal choice of regularization parameters that minimize the difference to the phantom.

\begin{tabular}{|c|c|c|c|c|}
\hline & Gaussian & Peaked & Hollow & Complex \\
\hline Method & \multicolumn{4}{|c|}{$\delta(\%)$} \\
\hline$\overline{\text { optimum }}$ & $(1.3)$ & $(3.6)$ & $(4.6)$ & (10.7) \\
\hline $\mathrm{GCV}$ & 1.4 & 5.9 & 5.2 & 11.2 \\
\hline PRESS & 1.9 & 5.8 & 5.8 & 11.6 \\
\hline $\mathrm{DP}$ & 1.3 & 6.7 & 8.9 & 12.8 \\
\hline $\mathrm{AIC}_{\mathrm{C}}$ & 1.3 & 6.9 & 7.5 & 12.7 \\
\hline
\end{tabular}

\section{F. Comparison of the regularization methods using artificial data}

A common way to assess the performance of a tomography method is to reconstruct phantom profiles. We have prepared a set of phantoms with increasing complexity, changing from a simple Gaussian function to a very peaked one, sharply hollow one and finally to a complex hollow-peaked asymmetric profile as is shown in a upper row of Fig. 5. The reconstructions were evaluated with a resolution of $100 \times 150$ using the anisotropic MFI regularization. The added noise level, based on real discharge noise, was on average equal to $2 \%$ of the signals in each LOS.

The performance of the methods are summarized in Tab. II and a reconstruction using the GCV method is shown in the lower row of Fig. 5. The differences between the various regularization methods, applying them to all of the artificial profiles, were insignificant, because each regularization was always close to the optimal value. Therefore, in Fig. 5 only the reconstructions from GCV are depicted. The difference between the phantoms and the reconstructions are most obvious on the sharp edges of the hollow and complex profile, which are smoothed in the reconstruction due to a finite spatial resolution of the diagnostic. Generally, DP and $\mathrm{AIC}_{\mathrm{C}}$ provide slightly over-smoothed solutions with a higher residuum. The diversity of the methods on the real measurements is substantially larger then on the presented phantoms as will be shown in Sec. VID.

\section{RECONSTRUCTION UNCERTAINTY}

A common question of experimentalists related to tomography is how to estimate the uncertainty of the reconstruction and the derived quantities. The answer is complicated by the significant contribution of the regularization bias. The contribution of the bias and variance to the relative mean square error (MSE) of the phantom profile is shown in Fig. 6. Obviously, the bias can repre- 

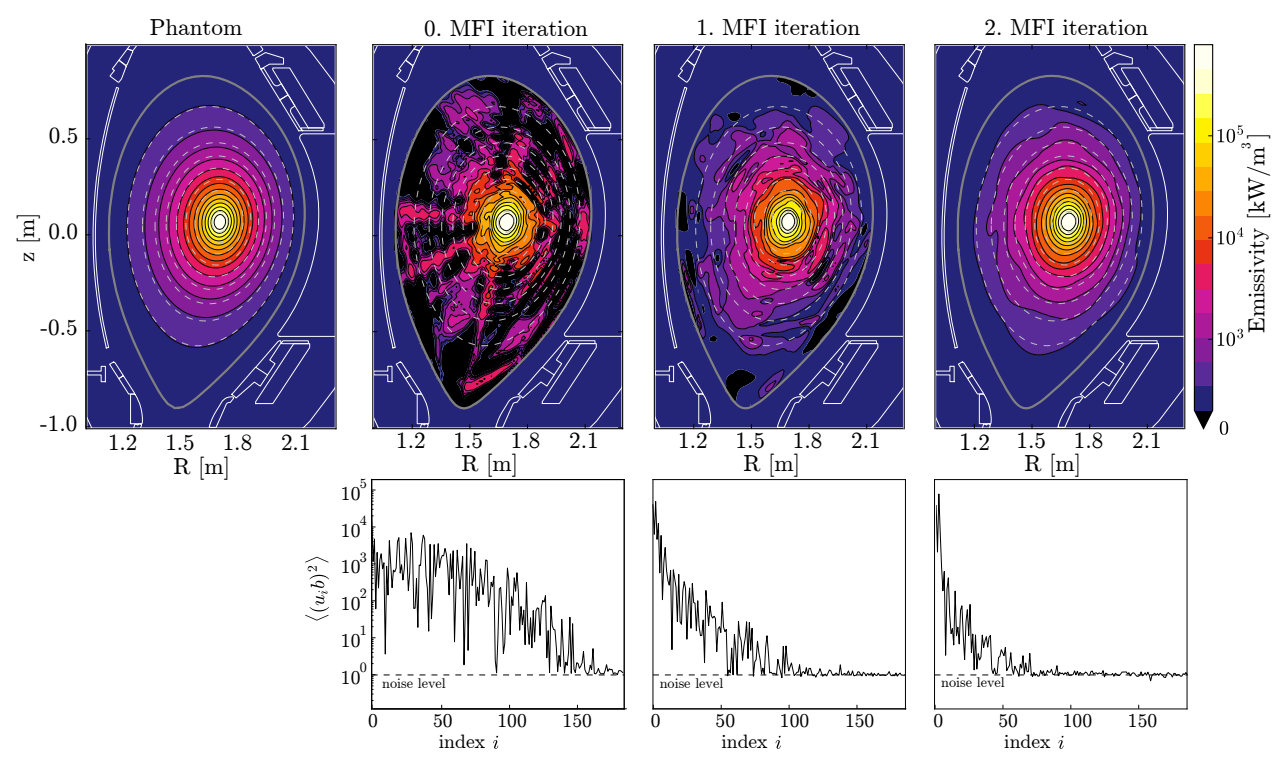

Figure 4. A tomographic reconstruction of a peaked radiation profile illustrating the effect of the anisotropic MFI iterations on the reconstruction quality. In the upper row the phantom profile and the reconstruction for 0 -th to 2-th MFI iterations in a nonlinear color-scale are shown. In the lower row the corresponding energy spectrum $\left(\mathbf{U}^{T} \mathbf{b}\right)_{i}^{2}$ is presented.
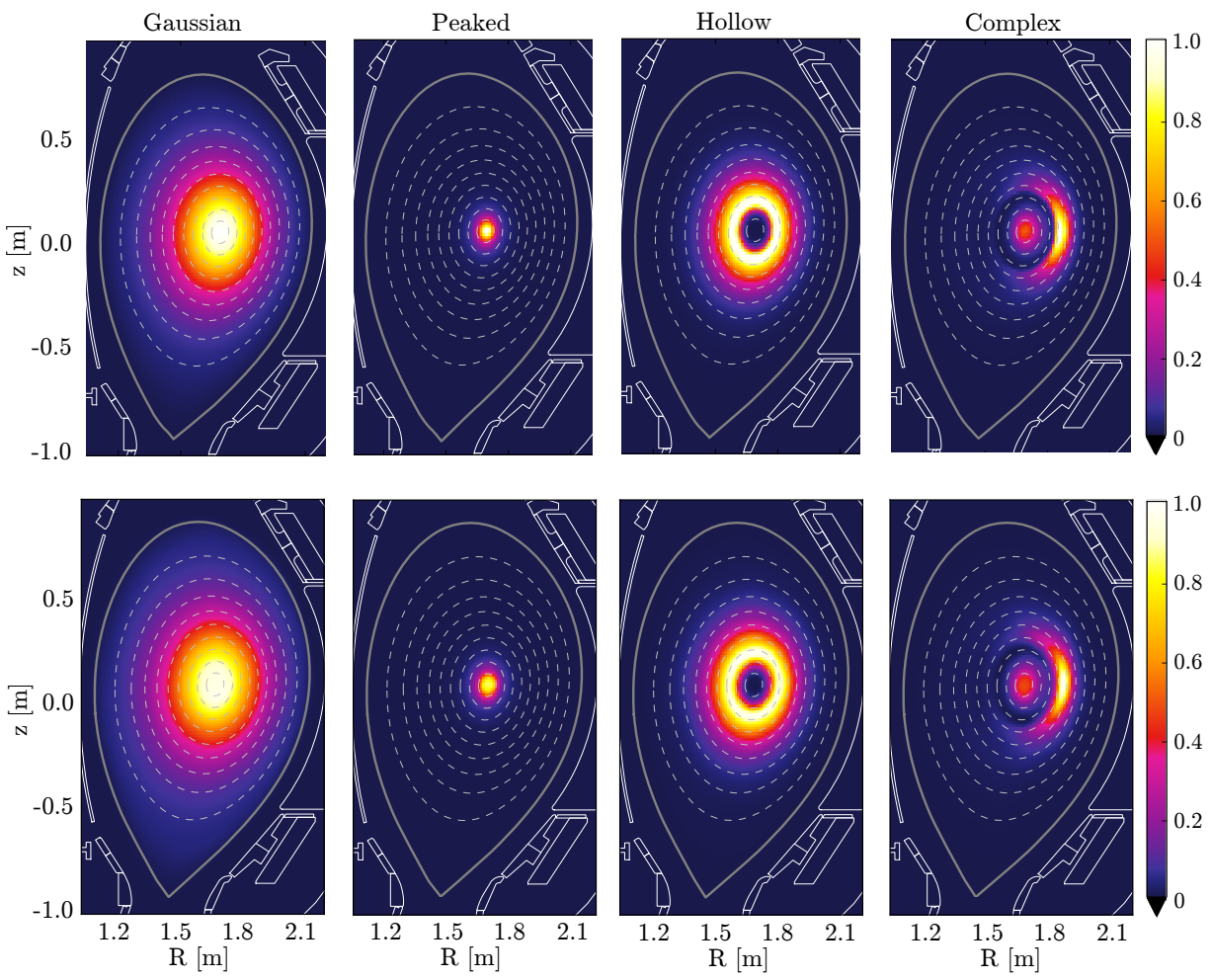

Figure 5. (upper row) Four phantoms used to evaluate the properties of the regularization selection methods. (lower row) The reconstruction of the phantoms with the regularization level estimated by GCV, which have always been very close to the optimal regularization level. All profiles are shown in the same color scale as the phantom profiles.

sent the majority of the MSE. In this section, the components of the MSE will be examined and an analytical formula for the covariance matrix of the solution will be proposed. Additionally, we will describe a way to treat the systematical errors. 

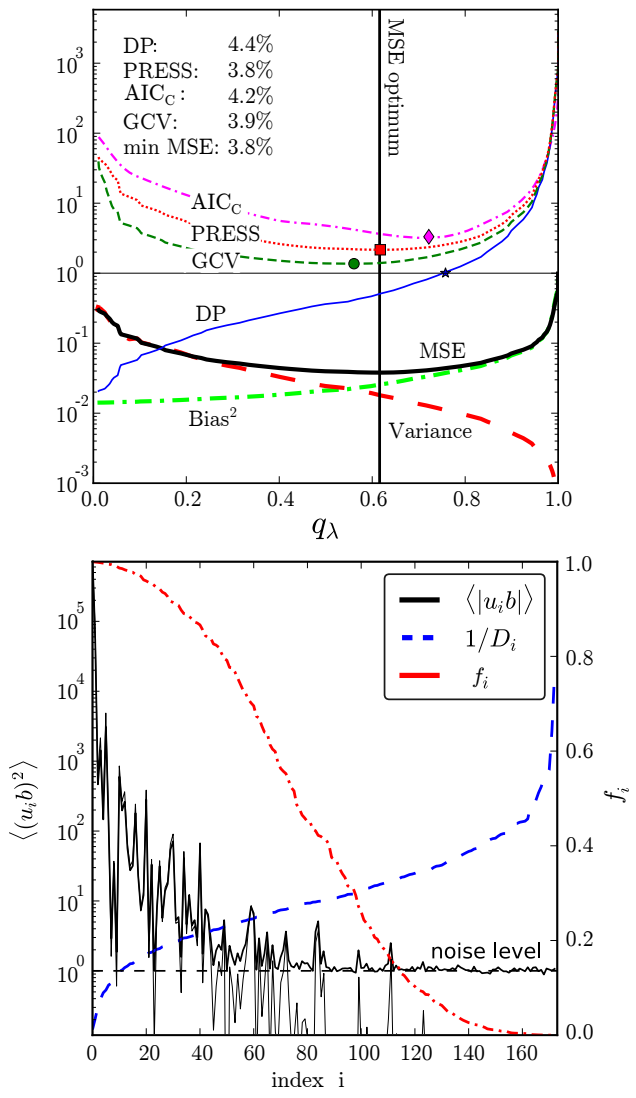

Figure 6. (top) The score of the GCV, PRESS, $\mathrm{AIC}_{C}$ and DP are plotted as a function of the regularization level $q_{\lambda}$. The minima of these functions and the root for DP are indicated by colored markers. The relative MSE obtained by comparison by the phantom with the reconstruction is shown by the black thick line. Two components of the MSE - the variance and bias are then indicated by a red dashed and green dashdotted line, respectively. The vertical black line indicates the position of the MSE minimum. (bottom) The second figure shows the mean energy spectrum $\left\langle\left(\mathbf{U}_{i} \mathbf{b}\right)^{2}\right\rangle$ of the measured data averaged over time (black thick line) and spectrum of noiseless data (black thin line). The GCV optimal filter factors $f_{i}=\left(1+\lambda / D_{i}^{2}\right)^{-1}$ are plotted in red and blue dashed indicates the unfolding amplification factor $1 / D_{i}$.

\section{A. Statistical and regularization error}

The regularized solution of Eq.(7) yields the following expression for the mean square error (MSE) in $\mathbf{g}_{\lambda}$ :

$$
\begin{gathered}
\left\langle\left(\mathbf{g}_{\lambda}-\mathbf{g}^{*}\right)^{2}\right\rangle_{k}=\sum_{i=1}^{r} f_{i, \lambda}^{2} \frac{\mathbf{V}_{k i}^{2}}{D_{i}^{2}}+ \\
+\left(\sum_{i=1}^{m}\left(1-f_{i, \lambda}\right) \frac{\mathbf{U}_{i}^{T} \mathbf{b}^{*}}{D_{i}} \mathbf{V}_{k i}+\left(\left(\mathbf{I}_{n}-\mathbf{V} \mathbf{V}^{T}\right) \mathbf{g}^{*}\right)_{k}\right)^{2}
\end{gathered}
$$

where $\langle\cdot\rangle$ indicates averaging over the fluctuating part, $\mathbf{g}^{*}$ is the accurate, but unknown, radiation profile and $\mathbf{b}^{*}=\mathbf{T g}^{*}$ is the accurate measurement, also unknown. The first term on the right side describes the variance caused by statistical uncertainty in the data. The error distribution is assumed to have zero mean and a standard deviation normalized to one. The term in the brackets represents the bias of our solution. The left term is the regularization error caused by suppressing small features in the measurement $\mathbf{b}^{*}$. Finally, the last term is a consequence of the rank deficiency of the projection matrix $\mathbf{T}$ and we will call it singularity error. Although the regularization error and the singularity error cannot be estimated without knowledge of the real radiation profile $\mathbf{g}^{*}$ and the brightness $\mathbf{b}^{*}$, we can perform a test on a class of the expected radiation profiles to estimate the influence of the bias on the reconstructed quantities. When only a weak regularization is applied, all filtering factors $f_{i}$ will be close to one and the statistical error will dominate the overall error. On the other hand, if strong regularization is used, most of the $f_{i}$ values vanish, and the error is dominated by the regularization error. The singularity error can be reduced only by including a priori knowledge more consistent with the solution (like anisotropic smoothing) and by better angular and spatial diagnostic coverage of the projection space (cf. Fig. 2).

The resolution of our reconstruction was assumed to be high enough to keep the pixel discretization error negligible. Moreover, the systematic errors in the geometry of the LOSs, their calibration, and other imperfection in the model were not included, because they can be significantly reduced as will be shown in Sec. VIC. Finally, the regularization parameter $\lambda$ is subject to statistical uncertainty as well. Nevertheless, close to the optimal regularization level, i.e the value of $\lambda$ minimizing MSE (see Fig. 6), the total MSE is only weakly sensitive to the perturbations in $\lambda$.

The statistical uncertainty in the reconstructed profile can be assumed as a lower bound for the real uncertainty. Based on the standard uncertainty propagation approach, the covariance matrix of the solution $\mathbf{g}_{\lambda}$ is given by

$$
\Sigma_{i j}^{g}=\left\langle\left(g_{i}-\left\langle g_{i}\right\rangle\right)\left(g_{j}-\left\langle g_{j}\right\rangle\right)\right\rangle=\sum_{k=1}^{r} f_{k}^{2} \frac{V_{i k} V_{j k}}{D_{k}^{2}} .
$$

where $\mathbf{V}$ and $\mathbf{D}$ are matrices introduced in the sSVD and sQR methods. Our tests of the phantom reconstructions indicate that the statistical variance is usually about half of the total MSE. Fig. 7a shows a zoom-in of a reconstruction of an artificial profile. The variances $\Sigma_{i i}^{g}$ associated with each pixel $i$ can be imaged as is demonstrated in Fig. 7b. Further, Fig. 7c shows the correlation of a single pixel indicated by the black cross with the neighboring pixels determined from the corresponding row of the covariance matrix. The width and shape of the correlation peak provide an estimate of the local spatial resolution.

\section{B. Discretization error}

The linear complexity of the decomposition methods allows for the calculation of the reconstructions on a very 

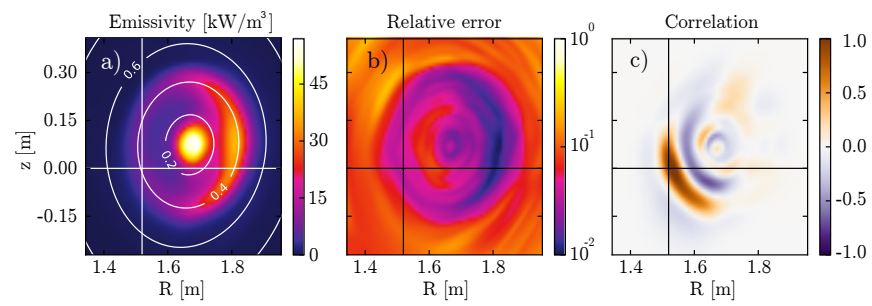

Figure 7. a) The SXR emissivity in the plasma core reconstructed from a phantom by the anisotropic MFI method and GCV regularization. The white contours indicate the normalized poloidal flux. b) The relative standard deviation $\delta_{i}=\sqrt{\left\langle g_{i}^{2}-\left\langle g_{i}\right\rangle^{2}\right\rangle} /\left\langle g_{i}\right\rangle$ of the reconstruction is shown. This is estimated from the diagonal of the covariance matrix $\sqrt{\Sigma_{i i}} / g_{i}$. c) The correlation of the pixel $j$, indicated by the black cross, to the surrounding pixels determined from the i-th row of the covariance matrix $\Sigma_{j i} / \Sigma_{j j}$. The same pixel $j$ is also marked in (a) and (b) of the figure.

fine grid. However, since only $\sim 200$ measurements are available, one could expect that increasing the resolution above some limit will lead only to smoother reconstructions without increasing their accuracy. This limit should depend on the size of the smallest features visible to the diagnostics. In order to tackle this question, we will examine the reconstruction error as function of the grid size. First, the brightness of a phantom is evaluated with a resolution of $200 \times 300$ pixels to minimize the discretization error. Then a tomographic reconstruction was performed for various reduced resolutions after adding 2\% noise. Finally, the solution was interpolated by a $2 \mathrm{D}$ spline to the original grid and compared with the high resolution phantom.

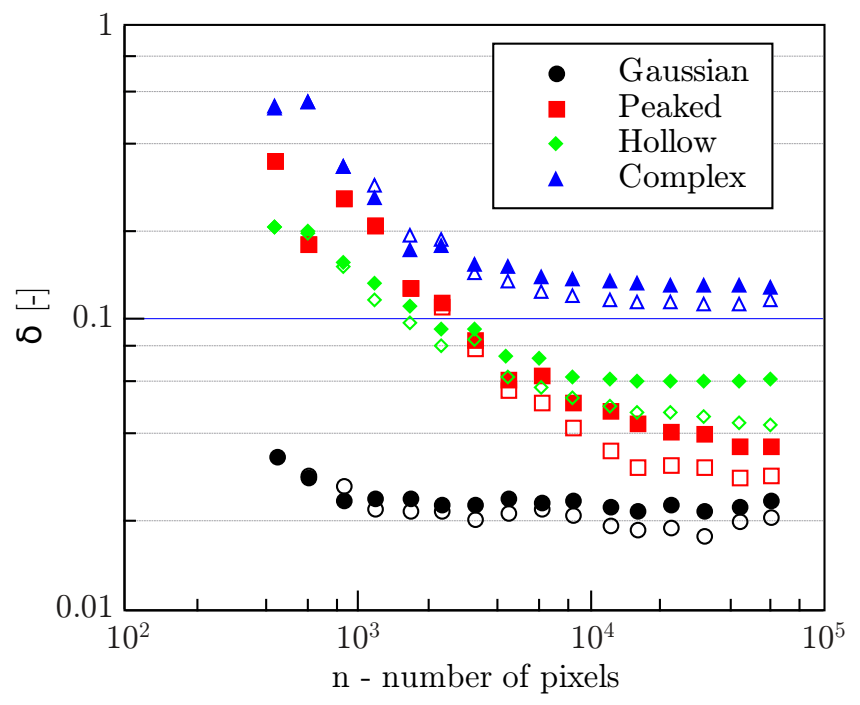

Figure 8. The dependence of the relative deviation $\delta$ (Eq. (19)) versus the number of pixels $n$ is presented. The full symbols correspond to $2 \%$ noise added to the artificial data while the open symbols are without noise.
The reconstruction errors for the four phantoms introduced earlier are shown in Fig. 8. The simple Gaussian profile can be well described already with $n \sim 1000$ pixel. But the reconstruction of sharp features in the other profiles requires a higher resolution, up to $n \sim 10^{4}$ pixels. The number of pixels necessary for the reconstruction can be reduced by using a variable grid size and local B-spline basis instead of pixels as was shown in Ref. 24 and 31 .

\section{Systematic errors}

The unavoidable limitation of the experimentally achievable accuracy is given by the systematical uncertainties in the geometry and calibration of the SXR cameras. Small deviations of the diode positions with respect to the camera slit in combination with a short focal length have a strong influence on the LOS positions and their etendue. The deviation of a diode position of a mere $0.2 \mathrm{~mm}$ leads to a roughly $2 \mathrm{~cm}$ shift of the LOS in the plasma core and consequently up to $20 \%$ discrepancies in the measured brightness in the regions of the steep SXR radiation.

The position and calibration errors may be diagnosed within the multi-camera SXR system ${ }^{10,11,58,59}$, because a large number of viewing angles provide sufficient redundancy of the measurements and the discrepancies can be identified and corrected. In the following the corrections applied in the present work are presented.

\section{Relative cross-calibration of the sensitivity}

The relative calibration of the SXR cameras at AUG was evaluated for every discharge in an iterative manner. First, the initial calibration factors were used to perform a reconstruction of the whole discharge. Then, a single correction factor for each camera was obtained in order to minimize the deviation between measure data and back-projected brightness. This procedure was repeated until convergence, which was typically achieved in the first step. Tests on the artificial profiles indicate that the cross-calibration can provide estimates with an accuracy well below $0.1 \%$. The evolution of the cross-calibration factors for five experimental campaigns is shown in Fig. 9. The scatter for the core heads is rather low, about 1$2 \%$ within a single campaign. Higher variations are observed in J3 and K1 during the first examined campaign probably caused by a deviation of the Be filters thickness, which was retrospectively measured to be up to $20 \mu \mathrm{m}$ different from the designed thickness of $75 \mu \mathrm{m}$. The higher scatter found in the side heads (shown green in Fig. 9) is caused by a low signal level, residual errors in the positioning of these cameras and uncertainties in the sensitivity and etendue of the off-axis LOSs. Neutron degradation ${ }^{60}$ may also be present since the total fluence over the AUG campaign is about $5 \cdot 10^{16}$ neutrons $/ \mathrm{m}^{2}$. 
However, no signatures of the neutron damage in the relative calibration were observed.
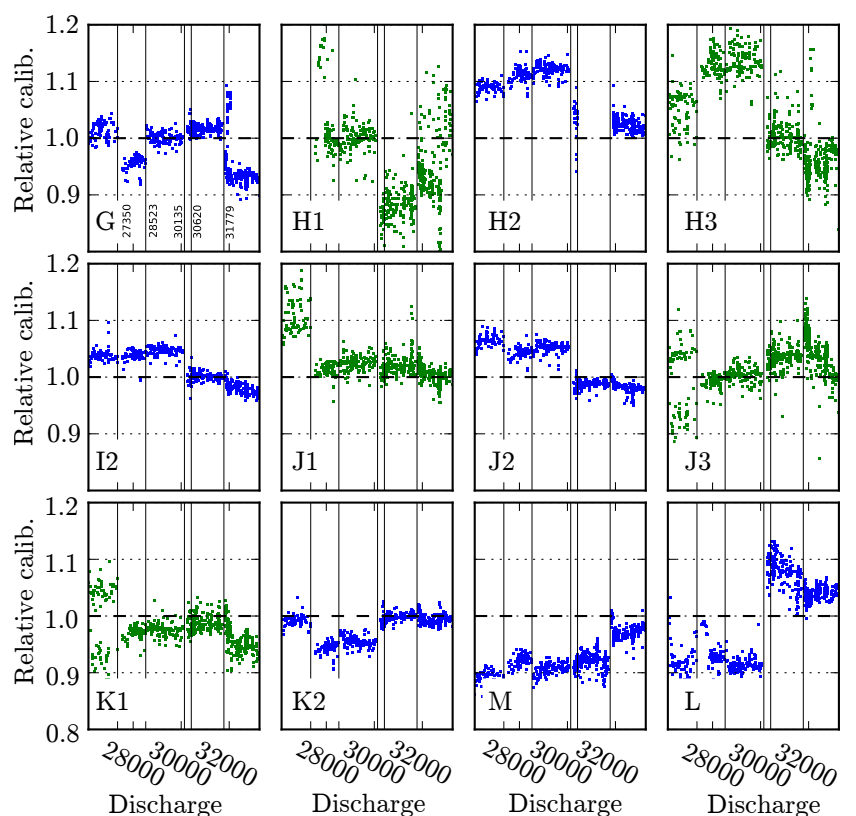

Figure 9. Relative calibration factors for each of 12 regularly used SXR camera heads evaluated for 758 discharges between 2011 and 2016; the blue points indicate factors for core heads, while the green correspond to the side heads. Vertical lines indicate vents of the chamber, replacing of the diodes arrays or Be filters and an extension of the viewing angles for cameras G, L and M.

\section{Adjustments of the viewing geometry}

The self-consistent calibration of the head positions was obtained in a set of discharges with negligible centrifugal asymmetries and significant variation in the peaking of the radiation profile. The shape of the magnetic flux surfaces was verified by a comparison between the CLISTE code ${ }^{61}$ and the TRANSP equilibrium solver ${ }^{62}$ constrained by the kinetic profiles. The deviation in the position of the flux-surfaces in the selected discharges was smaller than $5 \mathrm{~mm}$. The optimal position correction was identified by a gradient descent algorithm iteratively modifying the poloidal tilt for each of the 13 heads, to minimize the residuum between the measured brightness and the back-projection. New crosscalibration factors matching the actual geometry had to be estimated in every step. The regularization level was fixed to reduce the number of the nested optimization loops and increase the stability. Additionally, since no poloidal asymmetries were expected in the selected discharges, the solution was kept constant on the flux surfaces by the projection matrix $\mathbf{P}$ introduced in the section III B. In the end, the optimal tilt was estimated as a median over the set of tested discharges. The variation in the tilt corrections was about $0^{\circ} 3^{\prime}$ for the core heads and $0^{\circ} 12^{\prime}$ for the side heads. The estimated tilts corrections from campaign 2015 are specified in the following table:

\begin{tabular}{lccccccc}
\hline \hline Camera & $\mathrm{F}$ & $\mathrm{G}$ & $\mathrm{H} 1$ & $\mathrm{H} 2$ & $\mathrm{H} 3$ & $\mathrm{I} 2$ & \\
\cline { 2 - 7 } Tilt $\left[^{\circ}\right]$ & -0.26 & -0.99 & -0.68 & -0.46 & 0.10 & -0.82 & \\
Camera & $\mathrm{J} 1$ & $\mathrm{~J} 2$ & $\mathrm{~J} 3$ & $\mathrm{~K} 1$ & $\mathrm{~K} 2$ & $\mathrm{~L}$ & $\mathrm{M}$ \\
\cline { 2 - 7 } Tilt $\left[^{\circ}\right]$ & -1.43 & -1.28 & 1.12 & -1.59 & -0.32 & 1.67 & 1.23 \\
\hline \hline
\end{tabular}

No significant changes in these correction factors were observed during the campaign of 2015. However, manipulation of the cameras during the maintenance events of tokamak chamber has often resulted in small position deviations. The proper cross-calibration and self-consistent positioning of the camera heads has reduced the reconstruction residua by an order of magnitude. Further, the suppression of the systematic errors has significantly enhanced the stability and reliability of all regularization selection methods when used on the experimental measurements. Finally, the measurement accuracy of the small stationary poloidal asymmetries in the SXR radiation was also significantly improved.

\section{Robustness of the regularization methods for real datasets}

The crucial criterion for the choice of the optimal regularization method is their reliability and robustness for the real measurements. All regularization methods have shown an excellent performance on the artificial profiles; the failure rate was zero, and the estimated regularization had never been far from the optimal value minimizing MSE. However, the real data present additional challenges. We have performed a test on 100 randomly chosen discharges representing together 130000 time-points. In contrast to the phantom based tests the original emissivity, called also ground truth, is unknown. Therefore, our investigation aims to identify the number of clearly overor under-regularized tomograms, where the regularization method has failed to identify a meaningful solution. The comparison between the regularization methods is presented in Tab. III. The most robust is the PRESS method, working in $99.85 \%$ of the analyzed time-points. Since the GCV regularization is less conservative, the regularization level is usually lower and suffers from a higher number of under-regularized time-points. Most of the failures occurred in time-points with a low signal to noise ratio (SNR), while a performance similar to the PRESS method was observed in the hotter plasmas with a better SNR, which are usually considered for the SXR tomographic reconstructions. Finally, the DC and $\mathrm{AIC}_{\mathrm{C}}$ methods provides a comparable level of regularization that is even higher than the estimate from PRESS. The advantage of $\mathrm{AIC}_{\mathrm{C}}$ is higher stability and lower predisposition for extreme values of the regularization parameter than DC. Nevertheless, both these methods are 
still limited by considerable uncertainty in the estimation of the real level of the noise in the signal.

Table III. The results of the stability test performed with various regularization methods on 130000 frames corresponding to 100 real plasma discharges. The first two columns contain the fraction of over- and under-regularized frames and the last column is the average difference of the regularization level $q_{\lambda}$ with respect to the PRESS method.

\begin{tabular}{|c|c|c|c|}
\hline Method & over-fitted & over-smoothed & $\left\langle\Delta q_{\lambda}\right\rangle$ \\
\hline$\overline{\mathrm{GCV}}$ & $6 \%$ & $0.1 \%$ & $-15 \%$ \\
\hline PRESS & $0.1 \%$ & $0.05 \%$ & $-\%$ \\
\hline $\mathrm{DC}$ & $3 \%$ & $19 \%$ & $13 \%$ \\
\hline $\mathrm{AIC}_{\mathrm{C}}$ & $0.4 \%$ & $10 \%$ & $13 \%$ \\
\hline
\end{tabular}

\section{POLOIDAL ASYMMETRIES OF THE SXR RADIATION}

The purpose of this section is not to provide an extensive overview of all poloidal asymmetries observed at AUG or introduce new physics; the aim is to demonstrate the capabilities of the new tomographic procedures introduced at ASDEX Upgrade. Recent studies of the poloidal asymmetries accomplished at the Alcator C-Mod tokamak (see Refs. 63 and 64) motivated an increased interest in the measurements of the impurity distribution on the flux surfaces. Well understood physics and sufficiently accurate measurements could provide valuable information about the fast particle distribution, plasma composition and the plasma position because all these quantities have a strong impact on the observed asymmetry. The stationary poloidal asymmetry can be characterized by the first angular Fourier components of the stationary emissivity on the magnetic flux surface

$$
g(\rho, \theta)=a_{0}(\rho)\left(1+a_{c}(\rho) \cos (\theta)+a_{s}(\rho) \sin (\theta)+\ldots\right),
$$

where $\rho$ and $\theta$ represent the radial coordinate and the geometrical poloidal angle, $a_{c}$ will be referred to as $i n$ out and $a_{s}$ as up-down asymmetry. In-out asymmetries in the poloidal distribution of high-Z impurities are commonly produced by the centrifugal force ${ }^{65}$, and in special cases also by fast particles with a highly anisotropic distribution function ${ }^{63}$. The neoclassical friction force $^{64}$ is expected to be responsible for the up-down asymmetry.

The capabilities of the developed tomographic procedure will be demonstrated for plasma discharge \#30812 at 4.40 and $4.65 \mathrm{~s}$. These two cases were selected for the analysis because of a significant variation in the poloidal asymmetry caused by the centrifugal force in the first timepoint and by the trapped minority ions produced by the ion cyclotron resonance heating (ICRH) in the second case. Additionally, both cases were selected shortly before a sawtooth crash when the peaking of the kinetic profiles and of the $\mathrm{W}$ density are maximal.
At $4.40 \mathrm{~s}$, the deuterium plasma was heated by $2.5 \mathrm{MW}$ of neutral beam injection (NBI) and the core ion temperature reached $T_{i}=4.5 \mathrm{keV}$, the core electron temperature was $T_{e}=2.5 \mathrm{keV}$, the electron density was low at $3 \cdot 10^{19} \mathrm{~m}^{-3}$ and the deuterium Mach number (defined as $\left.M_{D}=\sqrt{m_{D} v_{\phi}^{2} /\left(2 k_{B} T_{i}\right)}\right)$ in the plasma core was equal to $\sim 0.33$. At $4.65 \mathrm{~s}$, an additional $4.3 \mathrm{MW}$ of ICRH power was applied (ICRH started at 4.60s), introduced with an outboard side resonance. The ion and electron temperature increased to $T_{i}=6.0 \mathrm{keV}$ and $T_{e}=4.1 \mathrm{keV}$, while the electron density stayed rather low at $4 \cdot 10^{19} \mathrm{~m}^{-3}$. The Mach number was equal to 0.21 in the core, causing a non-negligible centrifugal force. The estimated hydrogen minority concentration was roughly $5 \%$ and the effective ionic charge, $Z_{\text {eff }}$, was approximately 2.0. Since the measured $\mathrm{W}$ concentration was of the order of $10^{-3}$, more than $90 \%$ of the core SXR radiation was emitted by tungsten ions.
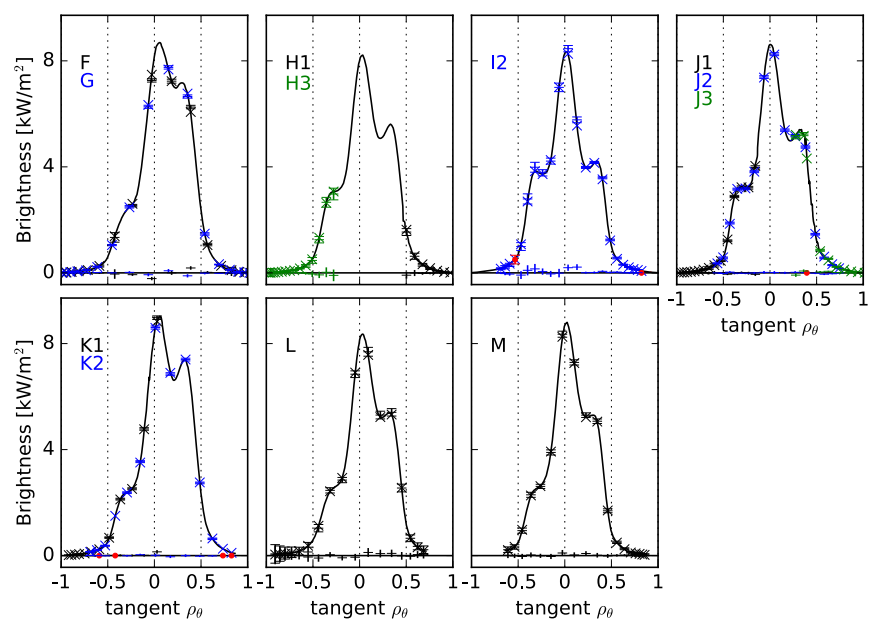

Figure 10. Depicted quantities are the measured brightnesses from the discharge \#30812@4.40s (horizontal crosses with error bars indicating estimated uncertainty), backprojections values from the tomographic reconstruction (diagonal crosses), cut through projection space in Fig. 2 averaged over the width of VOS (smooth black line), removed points (red dots) and crosses close to the zero line are residua between measurements and back-projections. The positive values of the normalized poloidal flux $\rho_{\theta}$ correspond to the outboard side of the plasma.

For $t=4.40 \mathrm{~s}$, the measured SXR brightnesses from all SXR LOSs are shown in Fig. 10. For the reconstruction, all available cameras were used except H2, which had with a broken biasing connector, and I1, I3, which had different Be filter thickness. The smooth black line with diagonal crosses indicates the back projected brightness from the tomographic reconstruction. Already the shape of the raw data suggests a complex profile with a significant outboard asymmetry. The corresponding tomographic reconstruction using sSVD method combined with GCV, evaluated at a resolution of $100 \times 150$ points is shown in Fig. 11a. The chosen pixel size of $13 \times 13 \mathrm{~mm}^{2}$ 
is safely below the scale of any features resolvable by the AUG SXR diagnostic. Figure 11 b shows a cut through the horizontal midplane of this profile, indicating an evident increase of the outboard SXR emissivity (blue) compared to the inboard emissivity (red). Finally, in Fig. 11c we have evaluated the centrifugal force $(\mathrm{CF})$ radiation asymmetry (red) as expected from $\mathrm{W}$ radiation and low$\mathrm{Z}$ ions bremsstrahlung. The model results are compared with the experimental observation (black).
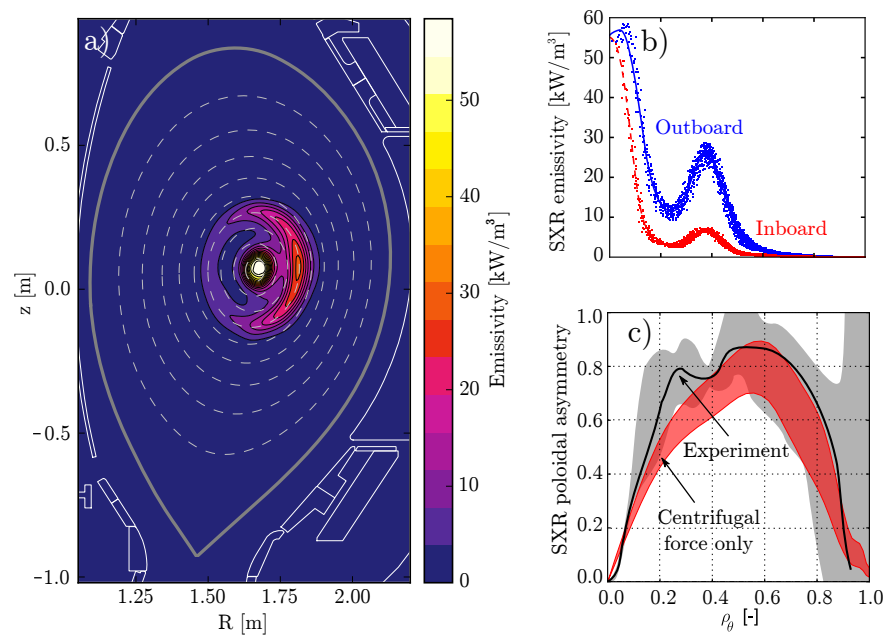

Figure 11. a) The tomographic reconstruction of SXR radiation profile from discharge \#30812 at 4.40 s evaluated at a resolution of $100 \times 150$ pixels with residuum $\chi^{2} / m=0.6$ and regularization level $q_{\lambda}=55 \%$ estimated by GCV is presented. b) The emissivity profile at the mid-plane is a cut through the tomogram (a), where the full blue line represents the outboard profile and the red dashed line the inboard profile. The scattered points indicate the presence of higher harmonics. c) Experimental asymmetry profile shown by the full black line with the gray confidence region is compared to the calculated CF asymmetry in red.

The confidence interval of the experimental asymmetry profile was estimated from the covariance matrix (Eq. 21) of the reconstruction and the assumption of $5 \mathrm{~mm}$ uncertainty in the plasma position. The magnetic equilibrium was obtained by the equilibrium solver in the TRANSP code constrained by the pressure profile. Both profiles of the asymmetry match reasonably well. The only significant deviation occurs at $\rho_{\theta}=0.25$ corresponding to the valley between the central peak and the outer ring, which is probably caused by the finite radial resolution of the tomography.

The SXR profile before the next sawtooth crash at $4.65 \mathrm{~s}$ is very different. Figure 12 shows the experimental data and an elevated level of inboard radiation in cameras F, G, H, L and M is already visible in the measured brightness profiles. The tomographic reconstruction is presented in Fig. 13a. The reconstruction of the emissivity distribution shows an inboard accumulation of $\mathrm{W}$ highlighted by a hollow radiation profile. Fig. 13b represents a mid-plane cut through the described emissivity profile. In this figure an additional outboard asymmetry in the outer regions of the plasma is visible. Finally, in Fig. 13c the experimentally obtained asymmetry is compared to the CF asymmetry, which describes the outboard region well. A clear deviation is present around the resonance position of the $\mathrm{ICRH}$, caused by the trapped minority ions. However, a detailed analysis and comparison with ICRH models goes beyond the scope of this work and will be addressed in future publications.

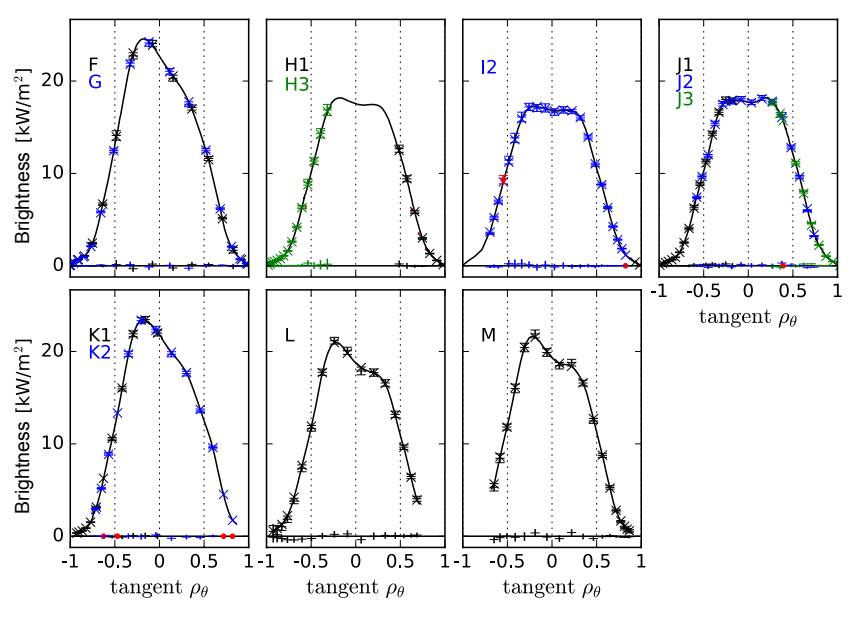

Figure 12. The SXR brightness obtained in discharge \#30812 at $4.65 \mathrm{~s}$ during the ICRH phase. Labelling and color scheme used in these plots is the same as in Fig. 10.
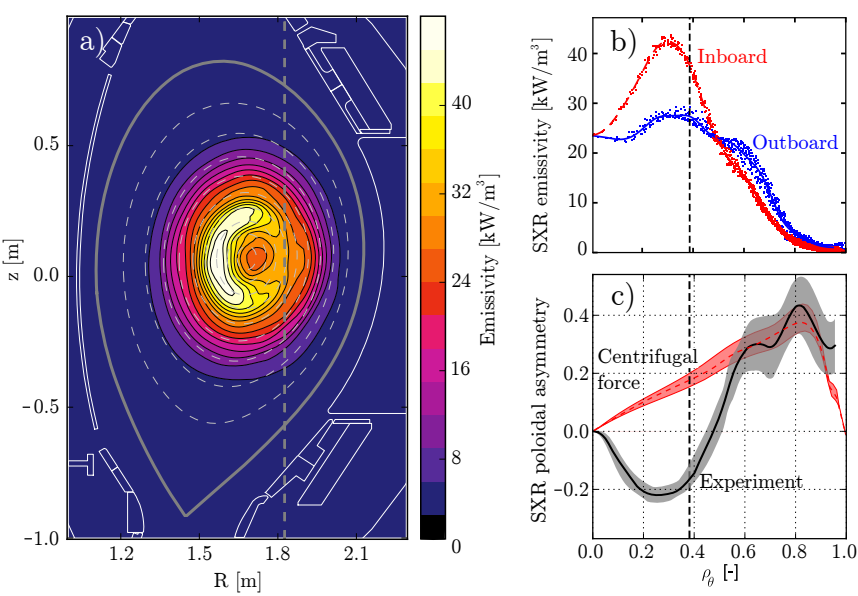

Figure 13. a) The reconstruction of the SXR radiation profile from discharge \#30812 at $4.65 \mathrm{~s}$ with the residuum $\chi^{2} / m=2.4$ and the regularization level $q_{\lambda}=49 \%$ determined by GCV is shown. The vertical dashed line indicates the position of the ICRH resonance. b) A midplane cut through the SXR emissivity profile shows a significant inboard accumulation of $\mathrm{W}$ in the core and outboard asymmetry in the outer regions. c) The asymmetry profile obtained from the tomographic reconstruction (black) is compared to the expected centrifugal asymmetry. 


\section{CONCLUSIONS}

We have described the modern multi-head SXR system of the ASDEX Upgrade tokamak (AUG). This diagnostic provides excellent coverage of the plasma with 208 lines of sight from 7 different viewing positions. In order to take advantage of this diagnostic also for the measurement of the stationary poloidal asymmetries in the SXR radiation, improvements in the precision of the measurement and the reconstruction algorithm were necessary.

We have shown that small deviations, e.g. in geometry, radiation filters thickness and amplification factors of the SXR diagnostic, represent a common limitation of the tomography reconstruction accuracy. Due to the high SXR radiation intensity from tungsten at $\mathrm{AUG}$, the statistical variance is often negligible compared to these systematical errors. We have presented a self-consistent calibration process involving a variation of the diagnostic geometry and a cross-calibration reducing residua between the data and the model by an order of magnitude. Also, the apparent reconstruction artifacts were suppressed, the spatial resolution was enhanced, and the stability of the regularization selecting methods was improved.

To access the full potential of this diagnostic, a new reconstruction algorithm was developed that performs a fast tomographic reconstruction with an excellent reconstruction quality. The inversion method was based on the generalized Tikhonov regularization minimizing the data fidelity and anisotropic Minimum Fisher information (MFI). First, multiple methods for the solution of this optimization problem were compared. The fastest reconstructions were obtained by the improved sparse QR method, followed closely by the sparse singular value decomposition (sSVD) method. Both algorithms have linear complexity in the number of pixels of the reconstruction, but the advantage of the sSVD method is the less complicated algorithm, more suitable for further development. The direct inversion, sparse generalized eigenvalues method, and generalized singular value method were substantially slower.

A critical issue of the Tikhonov regularization is the selection of the best regularization parameter. We have compared four methods - PRESS, $\mathrm{AIC}_{\mathrm{C}}, \mathrm{GCV}$, and DP, that are the most suitable for our problem. At first, the performance of these methods was investigated using artificial profiles where all methods provided an excellent estimate of the regularization parameter. However, tests on real data revealed significant differences. The most stable and reliable was the method based on the minimization of the predicted residual error sum of squares (PRESS). The stability of the generalized crossvalidation (GCV) is comparable to that of PRESS only for discharges with high signal to noise ratio (SNR). Occasionally the GCV minimum was not present, which led to significant over-fitting of the low SNR discharges. The unsatisfying performance of the discrepancy principle and the method based on the Akaike information criterion was probably caused by deviations in the estimate of the absolute uncertainty level in the real data. Additionally, we have proposed an analytical formula for the mean square error and the covariance matrix of the reconstruction. The covariance was used to determine the local statistical variance of the reconstruction and also to estimate the local spatial resolution of the tomography.

Finally, the performance of the AUG tomography was demonstrated on the reconstruction of two time slices of a discharge showing large poloidal asymmetries. In the first case, a low-density plasma heated by a neutral beam exhibits a significant centrifugal asymmetry that corresponds well to the value calculated from the parallel force balance. In the second case, the application of ion cyclotron heating on the outboard midplane led to a change in the asymmetry. Close to the resonance position a significant increase of the inboard SXR radiation occurred. The asymmetry, overcoming the still present centrifugal force, was caused by the trapped fast minority particles with a highly anisotropic temperature distribution. Since there are not many alternative diagnostics measuring highly energetic minority particles at the place of their origin, accurate measurements and a solid understanding of the physics of poloidal asymmetries can improve our knowledge about the fast ions.

\section{ACKNOWLEDGMENTS}

This work has been carried out within the framework of the EUROfusion Consortium and has received funding from the Euratom research and training program 20142018 under grant agreement No 633053. The views and opinions expressed herein do not necessarily reflect those of the European Commission.

\section{REFERENCES}

${ }^{1}$ H. Nakamura, S. Sakurai, S. Suzuki, T. Hayashi, M. Enoeda, K. Tobita, and D. P. D. Team, Fusion engineering and design 81(8), 1339 (2006).

${ }^{2}$ G. Janeschitz, JCT, ITER, et al., Journal of Nuclear Materials 290, 1 (2001).

${ }^{3}$ D. Meade, S. Jardin, J. Schmidt, et al., in Proceedings of the 18 th IAEA Conf. on Fusion Energy, Sorrento, Italy October (2000).

${ }^{4}$ T. Pütterich, R. Neu, R. Dux, A. Whiteford, M. O'Mullane, H. Summers, et al., Nuclear Fusion 50(2), 025012 (2010).

${ }^{5}$ R. Neu, R. Dux, A. Kallenbach, T. Pütterich, M. Balden, J. Fuchs, A. Herrmann, C. Maggi, M. O'Mullane, R. Pugno, et al., Nuclear fusion 45(3), 209 (2005).

${ }^{6}$ D. Vezinet, D. Mazon, D. Clayton, R. Guirlet, M. O'mullane, and D. Villegas, Fusion Science and Technology 63(1), 9 (2013).

${ }^{7}$ L. Ingesson, B. Alper, B. Peterson, and J.-C. Vallet, Fusion Science and Technology 53(2), 528 (2008).

${ }^{8}$ R. Granetz and P. Smeulders, Nuclear fusion 28(3), 457 (1988).

${ }^{9}$ K. Ertl, W. Von der Linden, V. Dose, and A. Weller, Nuclear Fusion 36(11), 1477 (1996).

${ }^{10}$ M. Anton, H. Weisen, M. Dutch, W. Von der Linden, F. Buhlmann, R. Chavan, B. Marletaz, P. Marmillod, and P. Paris, Plasma physics and controlled fusion 38(11), 1849 (1996). 
${ }^{11}$ L. Ingesson, B. Alper, H. Chen, A. Edwards, G. Fehmers, J. Fuchs, R. Giannella, R. Gill, L. Lauro-Taroni, and M. Romanelli, Nuclear fusion 38(11), 1675 (1998).

${ }^{12}$ M. Odstrcil, J. Mlynar, T. Odstrcil, B. Alper, A. Murari, and J. E. Contributors, Nuclear Instruments and Methods in Physics Research Section A: Accelerators, Spectrometers, Detectors and Associated Equipment 686, 156 (2012).

${ }^{13}$ T. Odstrcil, T. Pütterich, M. Weiland, M. Odstrcil, A. Gude, A. U. Team, et al., in 42nd EPS Conference on Plasma Physics, European Physical Society (2015).

${ }^{14}$ R. Neu, V. Bobkov, R. Dux, J. Fuchs, O. Gruber, A. Herrmann, A. Kallenbach, H. Maier, M. Mayer, T. Pütterich, et al., Physica Scripta 2009(T138), 014038 (2009).

${ }^{15}$ M. Sertoli, C. Angioni, R. Dux, R. Neu, T. Pütterich, V. Igochine, et al., Plasma Physics and Controlled Fusion 53(3), 035024 (2011).

${ }^{16}$ R. Dux, A. G. Peeters, A. Gude, A. Kallenbach, R. Neu, and A. U. Team, Nuclear Fusion 39(11), 1509 (1999).

${ }^{17}$ A. Gude, in Active Control of Magneto-hydrodynamic Instabilities in Hot Plasmas (Springer, 2015), pp. 53-104.

${ }^{18}$ T. Odstrcil, T. Pütterich, R. Bilato, W. Weiland, D. Mazon, A. Gude, et al., in 43. EPS P1.055, European Physical Society (2016).

${ }^{19}$ V. Igochine, A. Gude, et al., Hotlink based Soft X-ray Diagnostic on ASDEX Upgrade (IPP 1/338), Tech. rep., Max-PlanckInstitut für Plasmaphysik (2010), http://edoc.mpg.de/476537.

${ }^{20}$ M. Anton, M. Dutch, and H. Weisen, 22nd EPS (1995).

${ }^{21} \mathrm{M}$. Anton, M. Dutch, and H. Weisen, Review of scientific instruments 66(7), 3762 (1995).

${ }^{22} \mathrm{~A}$. Ramm and A. Katsevich, The Radon transform and local tomography (CRC press, 1996).

${ }^{23}$ M. Weiland, A. Gude, V. Igochine, M. Maraschek, H. Zohm, R. Bohle, R. Dux, K. Lackner, T. Odstrčil, and T. Pütterich, Plasma Physics and Controlled Fusion 57(8), 085002 (2015).

${ }^{24} \mathrm{D}$. Vezinet, V. Igochine, M. Weiland, Q. Yu, A. Gude, D. Meshcheriakov, M. Sertoli, E. M. Team, et al., Nuclear Fusion 56(8), 086001 (2016).

${ }^{25}$ F. Scholze, H. Rabus, and G. Ulm, Journal of Applied Physics 84(5), 2926 (1998).

${ }^{26}$ D. Vezinet, D. Mazon, and P. Malard, J. appl. Phys. 114(2), 023104 (2013).

${ }^{27}$ A. Tikhonov, in Soviet Math. Dokl. (1963), vol. 5, pp. 1035-1038.

${ }^{28}$ N. Iwama et al., in Image Processing: Theory and Applications (Elsevier Amsterdam, 1993), pp. 377-380.

${ }^{29}$ A. Wingen, M. Shafer, E. Unterberg, J. Hill, and D. Hillis, Journal of Computational Physics 289, 83 (2015).

${ }^{30}$ G. Fehmers, L. Kamp, and F. Sluijter, Inverse problems 14(4), 893 (1998).

${ }^{31}$ L. Ingesson, The Mathematics of some Tomography Algorithms used at JET, Tech. rep., Commission of the European Communities, Abingdon (United Kingdom). JET Joint Undertaking (2000).

${ }^{32}$ N. Terasaki, Y. Hosoda, M. Teranishi, and N. Iwama, Fusion engineering and design 34, 801 (1997).

${ }^{33}$ J. Bielecki, L. Giacomelli, V. Kiptily, M. Scholz, K. Drozdowicz, S. Conroy, T. Craciunescu, M. Kempenaars, and J. E. Contributors, Review of Scientific Instruments 86(9), 093505 (2015).

${ }^{34}$ P. Hansen, Numerical algorithms 6(1), 1 (1994).

${ }^{35}$ D. Mazon, D. Vezinet, P. Malard, D. Pacella, L. Gabelieri, A. Romano, V. Piergotti, T. Benedetto, F. Murtas, S. Dagabov, et al., Nuclear Instruments and Methods in Physics Research Section A: Accelerators, Spectrometers, Detectors and Associated Equip- ment 720, 78 (2013).

${ }^{36}$ J. Mlynar, S. Coda, A. Degeling, B. Duval, F. Hofmann, T. Goodman, J. Lister, X. Llobet, and H. Weisen, Plasma physics and controlled fusion $\mathbf{4 5}(2), 169$ (2003).

${ }^{37} \mathrm{P}$. Hansen, Inverse problems 8(6), 849 (1992).

${ }^{38}$ Y. Chen, T. Davis, W. Hager, and S. Rajamanickam, ACM Transactions on Mathematical Software (TOMS) 35(3), 22 (2008).

${ }^{39}$ G. H. Golub and C. F. Van Loan, Matrix computations, vol. 3 (JHU Press, 2012).

${ }^{40}$ Y. Hosoda and T. Torii, Jpn. J. appl. Math. 4, 287 (1994).

${ }^{41} \mathrm{R}$. Lehoucq, D. Sorensen, and C. Yang, ARPACK users, guide: solution of large-scale eigenvalue problems with implicitly restarted Arnoldi methods, vol. 6 (Siam, 1998).

${ }^{42}$ C. Van Loan, Numerische Mathematik 46(4), 479 (1985).

${ }^{43}$ D. Mazon, D. Vezinet, D. Pacella, D. Moreau, L. Gabelieri, A. Romano, P. Malard, J. Mlynar, R. Masset, and P. Lotte, Review of Scientific Instruments 83(6), 063505 (2012).

${ }^{44}$ V. Loffelmann, J. Mlynar, M. Imrisek, D. Mazon, A. Jardin, V. Weinzettl, and M. Hron, Fusion Science and Technology 69(2) (2016).

${ }^{45} \mathrm{M}$. Bertero, C. De Mol, and E. Pike, Inverse problems 4(3), 573 (1988).

${ }^{46} \mathrm{P}$. Hansen, SIAM review 34(4), 561 (1992).

${ }^{47}$ N. Iwama, H. Yoshida, H. Takimoto, Y. Shen, S. Takamura, and T. Tsukishima, Applied Physics Letters 54(6), 502 (1989).

${ }^{48}$ C. Hurvich and C. Tsai, Biometrika 76(2), 297 (1989).

${ }^{49} \mathrm{~T}$. Kitagawa, Japan Journal of Applied Mathematics 4(3), 371 (1987).

${ }^{50} \mathrm{~A}$. Tikhonov and V. Glasko, USSR Computational Mathematics and Mathematical Physics 5(3), 93 (1965).

${ }^{51}$ D. Girard, SIAM Journal on Scientific and Statistical Computing 8(6), 934 (1987).

${ }^{52}$ N. Iwama, IAEA Technical Committee Meeting (1990).

${ }^{53}$ G. Wahba, SIAM Journal on Numerical Analysis 14(4), 651 (1977).

${ }^{54}$ G. Golub, M. Heath, and G. Wahba, Technometrics 21(2), 215 (1979).

${ }^{55}$ N. Iwama, T. Tsuji, and Y. Hosoda, A bulletin of Daido Technical College 42, 93 (2006).

${ }^{56}$ H. Akaike, Automatic Control, IEEE Transactions on 19(6), 716 (1974).

${ }^{57}$ C. Hurvich, J. Simonoff, and C. Tsai, Journal of the Royal Statistical Society: Series B (Statistical Methodology) 60(2), 271 (1998).

${ }^{58}$ E. Lyadina, C. Tanzi, D. da Cruz, and A. Donne, in EPS, 17C(Part III) (1993).

${ }^{59}$ C. Görner, Tomographische Untersuchung von globalen AlfvénEigenmoden am Stellarator Wendelstein 7-AS, Ph.D. thesis, Max-Planck-Institut für Plasmaphysic (1998).

${ }^{60}$ R. Korde, A. Ojha, R. Braasch, and T. C. English, IEEE Trans. Nucl. Sci. 36(6), 2169 (1989).

${ }^{61} \mathrm{P}$. McCarthy et al., The CLISTE interpretive equilibrium code, Tech. rep., Max-Planck-Institut für Plasmaphysik (1999).

${ }^{62}$ J. DeLucia, S. Jardin, and A. Todd, Journal of Computational Physics 37(2), 183 (1980).

${ }^{63}$ M. Reinke, I. Hutchinson, J. Rice, N. Howard, A. Bader, S. Wukitch, Y. Lin, D. Pace, A. Hubbard, J. Hughes, et al., Plasma Physics and Controlled Fusion 54(4), 045004 (2012).

${ }^{64}$ M. Reinke, J. Rice, I. Hutchinson, M. Greenwald, N. Howard, J. Hughes, J. Irby, Y. Podpaly, J. Terry, and A. White, Nuclear Fusion 53(4), 043006 (2013).

${ }^{65} \mathrm{~J}$. Wesson, Nuclear fusion 37(5), 577 (1997). 